\title{
T-duality in gauged linear sigma-models with torsion
}

\author{
Dan Israël \\ Laboratoire de Physique Théorique et Hautes Énergies, Université Pierre et Marie Curie, \\ 4 place Jussieu, 75252, Paris Cedex 05, France ${ }^{1}$ \\ E-mail: israel@lpthe.jussieu.fr
}

ABSTRACT: $(0,2)$ gauged linear sigma models with torsion, corresponding to principal torus bundles over warped CY bases, provide a useful framework for getting exact statements about perturbative dualities in the presence of fluxes. In this context we first study dualities mapping the torus fiber onto itself, implying the existence of quantization constraints on the torus moduli for consistency. Second, we investigate dualities mixing the principal torus bundle with the gauge bundle, relating the torsional GLSMs to ordinary ones corresponding to CY compactifications with non-standard embeddings, namely two classes of models with different target-space topologies.

Keywords: Flux compactifications, Superstrings and Heterotic Strings, String Duality

ArXiv EPRINT: 1306.6609

\footnotetext{
${ }^{1}$ Unité Mixte de Recherche (UMR 7589) de l'Université Pierre et Marie Curie et du CNRS.
} 


\section{Contents}

$\begin{array}{llr}1 & \text { Introduction } & 1\end{array}$

2 Linear sigma-models for torsional compactifications 3

2.1 Torsional torus bundles in heterotic supergravity 3

$2.2(0,2)$ gauged linear sigma-models for K3 5

2.3 Torus fibration and torsion 6

2.4 Moduli quantization 8

3 Torus self-duality 11

$\begin{array}{lll}3.1 \text { Warm-up } & 11\end{array}$

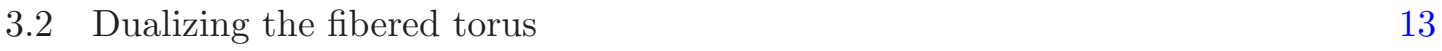

$\begin{array}{lll}3.3 & \text { Perturbative duality group } & 16\end{array}$

4 Torus/bundle dualities $\quad 17$

$4.1(0,2)$ bosonization of charged Fermi multiplets $\quad 17$

$\begin{array}{lll}4.2 & \text { Wilson lines } & 19\end{array}$

\begin{tabular}{ll}
4.3 Gauge-torus duality 20 \\
\hline
\end{tabular}

5 Conclusion $\quad 22$

A Two-dimensional $(0,2)$ superspace $\quad 23$

$\begin{array}{lll}\text { A.1 Superfields } & 23\end{array}$

$\begin{array}{lll}\text { A.2 } & (0,2) \text { Lagrangians } & 25\end{array}$

$\begin{array}{ll}\text { B Two-tori } & 27\end{array}$

\section{Introduction}

Gauged linear sigma models (GLSMs), introduced by Witten [1], are defined as $(2,2)$ or $(0,2)$ supersymmetric two-dimensional gauge theories with Abelian gauge groups, that are designed to flow in the infrared, in their geometric phase, to superconformal non-linear sigma-models suitable for supersymmetric string compactifications. They provide a very efficient tool for computing topological quantities in worldsheet theories for Calabi-Yau compactifications, even far away from the special loci in moduli space where a conformal field theory description is known; for a recent review see [2]. Recently, $(0,2)$ linear sigma models with torsion, describing heterotic compactifications with flux, triggered a renewal of interest for this subject [3-12]. GLSMs are even more useful in this context, since so little is known about the space of torsional compactifications. 
Moving away from the phenomenologically unappealing case of CY compactifications with standard embedding of the spin connection in the gauge connection is possible by changing the gauge bundle or adding three-form flux threading the compact geometry. These two aspects are actually tied together by the modified heterotic Bianchi identity, which proves notoriously arduous to solve. Explicit solutions of the supersymmetry equations at order $\alpha^{\prime}$ that were written down long time ago [13] appeared only rather recently. A class of solutions, consisting in principal torus bundles over a warped CY base, were first discovered using string dualities [14], then, following [15], shown to be provide actual solutions of the supersymmetry equations [16]. Other classes of solutions belong to a mostly uncharted territory. Indeed, having a non-trivial three-form flux results in the metric loosing Kählerity and being conformally balanced instead of Calabi-Yau [17, 18]. Some noncompact conifold solutions with torsion were nevertheless found in [19] as exact worldsheet CFT backgrounds, and supergravities generalizations thereof were studied recently in [20].

In order to deal with compact torsional heterotic backgrounds, gauged linear sigma models are the only available models at present. Adding torsion to $(0,2)$ GLSMs was first understood in [3-6], where the torus bundle solutions mentioned in the last paragraph were obtained by canceling the worldsheet gauge anomaly coming from the non-standard gauge bundle against classically non gauge-invariant axial couplings of chiral multiplets with a gauged shift-symmetry. Other types of models were subsequently discussed in $[8,9]$ where logarithmic axial couplings of ordinary charged chiral multiplets were used for the same purpose. Latter on it was realized [8-10] that these logarithmic terms can arise in certain branches of ordinary $(0,2)$ GLSMs, at one loop; thus, it seems that torsional GLSMs with log couplings are ordinary GLSMs in disguise. These results motivated a more general study of $(0,2)$ gauge theories initiated in [12].

Mirror symmetry [21] can certainly help in understanding the intimate relations between torsional and torsion-free compactifications, as it relates generically worldsheet theories whose target spaces have different topologies. Generalization of mirror symmetry to $(0,2)$ models was considered first in [22] and is not yet well-understood (see [23] for recent developments); including torsional GLSMs has not been done yet.

In this note, as a modest step in this direction, we investigate dualities between the gauged linear sigma-models with torsion corresponding to principal two-torus bundles over K3, equipped with some holomorphic gauge bundle $V$, and $(0,2)$ GLSMs for $T^{2} \times K 3$ with an additional line bundle over the K3 surface. Perturbative dualities of this sort have been already investigated from a target-space perspective [24-26], using the heterotic generalization of Buscher rules (for a review see [27]). It allowed to relate these seemingly distinct class of string backgrounds, however only at lowest order in $\alpha^{\prime}$; this is not really satisfactory in the present context as these solutions involve typically compactification at string scale. ${ }^{1}$ It was also argued in [30] that dualities of this sort allow to embed heterotic solutions in a higher-dimensional theory.

\footnotetext{
${ }^{1}$ In $[28,29]$, using an $\mathcal{M}$-theory construction, it was argued independently that there was a transition between these two types of compactifications at orbifold points of the K3 surface.
} 
Dualities mapping the torus fiber onto itself are also worthwhile to consider. We provide the mapping of parameters between the dual models, and show that supplementary quantization constraints on the moduli arise by demanding consistency of the dual theory for generic duality transformations. Interestingly, these conditions force the torus moduli to be those of a rational conformal field theory.

We will implement these two classes of dualities at the level of the gauged linear sigmamodel, showing that they are exact symmetries of the heterotic strings; some preliminary steps in this direction can be found in [5]. These dualities are actually symmetries of the GLSM themselves, even though the models could, in principle, be distinguished by irrelevant couplings that would not spoil the dualities between their respective infrared fixed points. Technically, we prove these dualities by introducing an extra worldsheet gauge field together with an extra chiral superfield, playing the role of a Lagrange multiplier, following closely the methods of [31]. ${ }^{2}$ For dualities mixing the torus and gauge bundles, we point out a subtlety related to the left-moving GSO projection, that would be hard to guess from a supergravity perspective. We also discuss the Wilson line moduli and their mixing with torus moduli.

Heterotic compactifications with line bundles attracted lot of attention recently, since they allow for computationally-efficient ways of getting standard-model-like spectra, the Hermitian-Yang-Mills equations being simple to solve [33]; they also appear naturally in GLSM descriptions of resolved heterotic orbifolds [34]. Hence, being able to map them to torsional solutions, consisting in principal torus bundles over a Calabi-Yau base, may shed new light on their properties and allow to embed them in a web of dualities involving type IIB or $\mathcal{M}$-theory compactification with fluxes.

This letter is organized as follows. In section 2 we review the construction of $(0,2)$ GLSMs for non-Kähler principal bundles over CY bases and make some important remarks about moduli quantization. In section 3 we study self-dualities between models within this category. In section 4 we obtain more general perturbative dualities, mixing the torus bundle with the gauge bundle. Finally in section 5 we discuss some implications of these results. Useful material is gathered in the appendices.

\section{Linear sigma-models for torsional compactifications}

In this section we summarize briefly the construction of gauged linear sigma-models with torsion discovered in [3], highlighting the most salient features for our present purposes, and discuss in detail the quantization of the torus moduli. We shall first review the geometry of the torsional compactifications that they correspond to.

\subsection{Torsional torus bundles in heterotic supergravity}

Heterotic compactifications to four-dimensions with $\mathcal{N}=2$ space-time supersymmetry are given by solutions of the type first obtained by Dasgupta, Rajesh, and Sethi using non-

\footnotetext{
${ }^{2}$ The same methods can be applied to the solvable worldsheet descriptions of non-compact models of this sort, found in [32], allowing to prove directly T-duality for superconformal theories.
} 
perturbative dualities [14] and discussed afterwards by many authors. They are given by principal two-torus bundles over a K3 base with Hermitian-Yang-Mills gauge bundles.

Let us discuss these $T^{2} \hookrightarrow \mathcal{C}_{6} \stackrel{\pi}{\rightarrow} K 3$ solutions explicitly. In our conventions, $U$ and $T$ correspond respectively to the generalized Kähler structure and to the complex structure, see appendix B. Thereafter we work in $\alpha^{\prime}=1$ units, i.e. such that the self-dual radius is equal to one. One considers a ten-dimensional string-frame metric of the form

$$
\mathrm{d} s^{2}=\eta_{\mu \nu} \mathrm{d} X^{\mu} \mathrm{d} X^{\nu}+\frac{U_{2}}{T_{2}}\left|\mathrm{~d} x^{1}+T \mathrm{~d} x^{2}+\pi^{\star} \mathfrak{w}^{n} \alpha_{n}\right|^{2}+e^{2 A(y)} \mathrm{d} s^{2}(\mathcal{S}),
$$

where $\mathrm{d} s^{2}(\mathcal{S})$ is a Ricci-flat metric on a K3 surface $\mathcal{S}$ and $e^{2 A}$ is a warp factor depending on the K3 coordinates only.

The torus fibration data is given by a set of complex topological charges $\mathfrak{w}^{n}$ belonging to the lattice $\mathbb{Z}+T \mathbb{Z}$ and a corresponding set of anti-self-dual two-forms on K3, that we write locally as $\Pi_{n}=\frac{1}{2 \pi} \mathrm{d} \alpha_{n}$, such that

$$
\Pi_{n} \in H^{2}(\mathcal{S}, \mathbb{Z}) \cap H^{1,1}(\mathcal{S}) .
$$

The two-form $\Pi=\mathfrak{w}^{n} \Pi_{n}$ should also be primitive w.r.t. the base, namely $J \wedge \Pi=0$, where $J$ is the Kähler form of K3. The two-forms $\Pi_{n}$ are, by construction, elements of the Picard group $\operatorname{Pic}(\mathcal{S})$. This puts some constraints on the complex structure of $\mathcal{S}$, since generically the Picard group is empty. ${ }^{3}$ Supergravity solutions with $\mathcal{N}=1$ supersymmetry in four dimensions can be obtained if some $\Pi_{n}$ 's have a $(2,0)$ component (see e.g. [35]), however there is no known GLSM for such compactifications.

The NS-NS three-form follows then from supersymmetry. Introducing the globally defined one-form $\theta=\mathrm{d} x^{1}+T \mathrm{~d} x^{2}+\pi^{\star} \mathfrak{w}^{n} \alpha_{n}$ the torsion is given by

$$
H=\star_{\mathrm{K} 3} \mathrm{~d} e^{2 A}-\frac{U_{2}}{T_{2}} \operatorname{Re}\left(\bar{\theta} \wedge \star_{\mathrm{K} 3} \mathrm{~d} \theta\right) .
$$

A supersymmetric gauge bundle is obtained as the pullback of a holomorphic gauge bundle $V$ on K3 satisfying the zero-slope limit of the Hermitian-Yang-Mills equation, namely

$$
\left.F^{2,0}=F^{0,2}=0, \quad J\right\lrcorner F=0 .
$$

On K3 it implies anti-selfduality, i.e. that the bundle $V$ corresponds to an anti-instanton background. For line bundles, once imposing Dirac quantization for the magnetic flux, these conditions are similar to those satisfied by the two-forms $\Pi_{n}$ specifying the principal torus bundle.

In addition, it is possible to add an Abelian gauge potential over the total space $\mathcal{C}_{6}$, that would reduce to a Wilson line for a product manifold $K 3 \times T^{2}$ :

$$
A=\alpha_{I} \mathfrak{T}^{I} \theta=\alpha_{I} \mathfrak{T}^{I}\left(\mathrm{~d} x^{1}+T \mathrm{~d} x^{2}+\pi^{\star} \mathfrak{w}^{n} \alpha_{n}\right),
$$

whose embedding in the commutant of the structure group of $V$ is specified by the commuting matrices $\mathfrak{T}^{I}$.

\footnotetext{
${ }^{3}$ For a generic algebraic surface, $\operatorname{dim}(\operatorname{Pic}(\mathcal{S}))=1$ while for a generic elliptically fibered K3, $\operatorname{dim}(\operatorname{Pic}(\mathcal{S}))=2$.
} 
As was shown by Fu and Yau [16], taking a solution (2.1)-(2.4) of the $\mathcal{N}=2$ supersymmetry conditions, and provided that the following tadpole condition holds

$$
\frac{U_{2}}{T_{2}} \int_{\mathrm{K} 3} \star_{\mathrm{K} 3} \Pi \wedge \bar{\Pi}-\operatorname{ch}_{2}(V)=24,
$$

there exists a solution of the Bianchi identity for the warp factor exp $2 A$, which is a function of the base coordinates. ${ }^{4}$

\section{$2.2(0,2)$ gauged linear sigma-models for $\mathrm{K} 3$}

We start with an old-fashioned $(0,2)$ gauged linear sigma-model whose infrared fixed point would be a heterotic non-linear sigma model with a K3 surface target-space, if an important consistency condition was not blatantly violated.

A $(0,2)$ GLSM is an Abelian $\mathrm{U}(1)^{r}$ two-dimensional supersymmetric gauge theory coupled to charged chiral and Fermi multiplets. For simplicity of presentation we choose a single $\mathrm{U}(1)$ gauge group; the generalization to $\mathrm{U}(1)^{r}$ is straightforward and will be given in places. We refer to appendix A.1 for superspace conventions and A.2 for the component expansion of the Lagrangian.

The GLSM contains a set of chiral superfields $\Phi_{I}$ of gauge charges $Q_{I}$, a set of Fermi multiplets $\Gamma_{a}$ of charges $q_{a}$ and the pair of gauge superfields $(\mathcal{A}, \mathcal{V})$ that is needed to formulate gauge theories in $(0,2)$ superspace. The full Lagrangian of the theory is of the generic form (summation over indices $I$ and $a$ is implied):

$$
\begin{aligned}
\mathcal{L}_{K 3}= & -\frac{i}{2} \int \mathrm{d}^{2} \theta^{+} \bar{\Phi}_{I} \mathcal{D}_{-} \Phi_{I}-\frac{1}{2} \int \mathrm{d}^{2} \theta^{+} \bar{\Gamma}_{a} \Gamma_{a}-\frac{1}{8 e^{2}} \int \mathrm{d}^{2} \theta^{+} \bar{\Upsilon} \Upsilon \\
& -\frac{\mu}{2} \int \mathrm{d} \theta^{+} \Gamma_{a} J^{a}\left(\Phi_{I}\right)+\frac{t}{4} \int \mathrm{d} \theta^{+} \Upsilon+\text { h.c. }
\end{aligned}
$$

where the gauge superfield-strength $\Upsilon$, which is a chiral superfield is given in eq. (A.25). The holomorphic functions $J^{a}\left(\Phi^{I}\right)$ in the chiral superfields play a role analogous to the superpotentials of more familiar $(2,2)$ theories.

A model specified by the Lagrangian (2.7) is gauge-anomalous for a generic choice of charges, since left- and right-handed fermions belong to different multiplets. Under a super-gauge-transformation, parametrized by a chiral superfield $\Xi$, the effective Lagrangian is shifted by ${ }^{5}$

$$
\delta_{\Xi} \mathcal{L}_{\text {eff }}=\frac{\mathfrak{A}}{8} \int \mathrm{d} \theta^{+} \Xi \Upsilon+\text { h.c. }, \quad \text { with } \quad \mathfrak{A}=Q_{i} Q^{i}-q_{n} q^{n} .
$$

Well-behaved ordinary GLSMs require the vanishing of this anomaly. In contrast, to formulate torsional GLSMs we shall be happy with any positive value of $\mathfrak{A}$, since (2.8) will eventually be cancelled by the gauge shift of the torus fibre Lagrangian. ${ }^{6}$

\footnotetext{
${ }^{4}$ Different choices of connection with torsion lead to different differential equations for the warp factor; some choices give simpler answers than others [36].

${ }^{5}$ Our convention is $\mathcal{S}_{\text {eff }}=\frac{1}{2 \pi} \int d^{2} x \mathcal{L}_{\text {eff }}$.

${ }^{6} \mathrm{We}$ do also require, in the full theory, the existence of non-anomalous global chiral symmetries, giving the right $\mathrm{U}(1) \mathrm{R}$-current and the left $\mathrm{U}(1)$ flavour-current, that will be respectively part of the infrared superconformal algebra and used in the GSO projection, since we want to obtain as an infrared fixed point a NSLM for a supersymmetric heterotic string compactification.
} 
For illustrative purposes, let us consider a realization of $\mathrm{K} 3$ as a quartic in $\mathbb{P}^{3}$. In order to describe the geometry of the compactification one takes four chiral superfields $\Phi_{i}$ of gauge charge $Q=1$ and a single Fermi superfield $\Gamma_{0}$ of charge -4 . These charges satisfy $\sum_{i} Q_{i}=Q_{\Gamma_{0}}$, which translates geometrically in the CY condition $\mathrm{c}_{1}(T)=0$. Associated with these superfields is a superpotential coupling of the form

$$
\mathcal{L}_{s}=\int \mathrm{d} \theta^{+} \Gamma_{0} G\left(\Phi_{i}\right)+\text { h.c. }
$$

where $G\left(\phi_{i}\right)=0$ is a quartic that defines the K3 surface carved out of the vacuum manifold $\mathbb{P}^{3}$ in the CY phase $r \gg 1$. The right-handed fermions are, as usual, sections of the tangent bundle.

Supplementing the K3 surface with a gauge bundle can be done in many ways. One considers for instance an extra set of $s+1$ Fermi multiplets $\Gamma_{a}$ of charge $q_{a}=+1$, satisfying the standard chirality constraint (i.e. $\overline{\mathfrak{D}}_{+} \Gamma_{a}=0$ ), and a chiral multiplet $P$ of charge $Q_{p}=-(s+1)$. The superpotential couplings associated with these multiplets take the form:

$$
\mathcal{L}_{w}=\int \mathrm{d} \theta^{+} P \Gamma_{a} J^{a}\left(\Phi_{1, \ldots, 4}\right)+\text { h.c. }
$$

where the holomorphic functions $J^{a}\left(\phi_{i}\right)$ are homogeneous polynomials of degree $s$, as requested in order to ensure classical gauge invariance.

In the CY phase, the bottom components of the Fermi multiplets are sections of (the pullback of) the rank $s$ holomorphic vector bundle $\mathcal{V}$ over K3 determined by the short exact sequence

$$
0 \longrightarrow \mathcal{V} \longrightarrow \bigoplus_{a=1}^{s+1} \mathcal{O}(1) \stackrel{J^{a}}{\longrightarrow} \mathcal{O}(s+1) \longrightarrow 0
$$

Since we did not impose the constraint $\mathfrak{A}=0$ for gauge anomaly-cancellation, the model hitherto defined does not make sense as a quantum theory.

\subsection{Torus fibration and torsion}

The main idea of the work [3] is to realize the Green-Schwarz mechanism at the gauged linear sigma-model level by canceling the quantum gauge non-invariance of the K3 GLSM against the classical gauge variation of another term in the action that will eventually describe the fiber of the principal $T^{2}$ bundle. We consider a generic two-torus whose moduli are given by the complex parameters $U$ and $T$, respectively the generalized Kähler structure and the complex structure, see appendix B for our conventions.

For this purpose we add a pair of chiral superfields $\Omega_{1,2}$, that complexify the two-torus coordinates, whose imaginary shift-symmetry is gauged. Explicit expressions in components are given in appendix A.1. The scalar fields of these multiplets parametrize a $\mathbb{C}^{\star} \times \mathbb{C}^{\star}$ that we wish to reorganize as $\mathbb{C} \times T^{2}$, by a change of complex structure in target space, in order to decouple the non-compact directions.

Generalizing the construction of [3] to generic torus moduli, including a constant B-field along the fiber $\left(B=U_{1} \mathrm{~d} x^{1} \wedge \mathrm{d} x^{2}\right)$, the Lagrangian associated with these chiral multiplets 
reads

$$
\begin{aligned}
L_{f}= & -\frac{i U_{2}}{8 T_{2}} \int \mathrm{d}^{2} \theta\left(\Omega_{1}+\bar{\Omega}_{1}+T_{1}\left(\Omega_{2}+\bar{\Omega}_{2}\right)+2\left(\mathfrak{w}_{1}+T_{1} \mathfrak{w}_{2}\right) \mathcal{A}\right) \times \\
& \times\left(\partial_{-}\left(\Omega_{1}-\bar{\Omega}_{1}+T_{1}\left(\Omega_{2}-\bar{\Omega}_{2}\right)\right)+2 i\left(\mathfrak{w}_{1}+T_{1} \mathfrak{w}_{2}\right) \mathcal{V}\right) \\
& -\frac{i}{8} U_{2} T_{2} \int \mathrm{d}^{2} \theta\left(\Omega_{2}+\bar{\Omega}_{2}+2 \mathfrak{w}_{2} \mathcal{A}\right)\left(\partial_{-}\left(\Omega_{2}-\bar{\Omega}_{2}\right)+2 i \mathfrak{w}_{2} \mathcal{V}\right) \\
& +\frac{i}{8} U_{1} \int \mathrm{d}^{2} \theta\left[\left(\Omega_{1}+\bar{\Omega}_{1}+2 \mathfrak{w}_{1} \mathcal{A}\right)\left(\partial_{-}\left(\Omega_{2}-\bar{\Omega}_{2}\right)+2 i \mathfrak{w}_{2} \mathcal{V}\right)-\right. \\
& \left.\quad-\left(\Omega_{2}+\bar{\Omega}_{2}+2 \mathfrak{w}_{2} \mathcal{A}\right)\left(\partial_{-}\left(\Omega_{1}-\bar{\Omega}_{1}\right)+2 i \mathfrak{w}_{1} \mathcal{V}\right)\right] \\
& -\frac{i h^{\ell}}{4} \int \mathrm{d} \theta^{+} \Upsilon \Omega_{\ell}+\text { h.c. }
\end{aligned}
$$

where the shift-symmetry of $\operatorname{Im}\left(\omega_{\ell}\right)$ is gauged, with integer charges $\mathfrak{w}_{\ell}$. At the same time, the chiral superfields $\Omega_{\ell}$ are coupled axially to the gauge superfields, through the FayetIliopoulos (FI) field-dependent term that appears the last line of eq. (2.12). The axial couplings $h^{\ell}$ need to be integers, in order for the action to be invariant under $\omega_{\ell} \sim \omega_{\ell}+2 i \pi$ for any value of the two-dimensional instanton number $n=-\frac{1}{2 \pi} \int F$.

The Lagrangian given by eq. (2.12) is classically non gauge-invariant, because of the axial coupling. Under a super-gauge variation, whose parameter is a chiral superfield $\Xi$, the Lagrangian is shifted by

$$
\delta_{\Xi} \mathcal{L}_{g}=\frac{1}{4} h^{\ell} \mathfrak{w}_{\ell} \int \mathrm{d} \theta^{+} \Xi \Upsilon+\text { h.c. }
$$

This classical gauge variation can be used to precisely cancel the gauge anomaly (2.8) of the K3 GLSM, hence implementing the Green-Schwartz mechanism on the worldsheet. The values of the couplings $h_{\ell}$ are then determined by quantum gauge-invariance of the whole theory:

$$
2 h^{\ell} \mathfrak{w}_{\ell}=q_{n} q^{n}-Q_{i} Q^{i} .
$$

This model can be generalized slightly by allowing Wilson lines; we defer this discussion to section 4.

As it stands, this model describes a non-compact principal bundle over K3. In order to decouple the non-compact part of the fiber, one needs to cancel the couplings between the fermion field $\mu_{-}$of the gauge multiplet and the fermions $\chi_{+, \ell}$ of the shift superfields $\Omega_{\ell}$. Otherwise, the supersymmetry variation of the former $\delta_{\epsilon} \mu_{-}=i \epsilon\left(D+2 i F_{01}\right)$ would prevent the decoupling from being compatible with worldsheet supersymmetry.

Since these fermionic interactions come from the terms proportional to $\mathcal{V} \Omega_{\ell}$ in superspace, one simply needs to choose the parameters of the model in order that these terms vanish altogether. Explicitly it amounts to set

$$
\begin{aligned}
\frac{U_{2}}{T_{2}}\left(\mathfrak{w}_{1}+T_{1} \mathfrak{w}_{2}\right)-U_{1} \mathfrak{w}_{2}+h^{1} & =0, \\
\frac{U_{2}}{T_{2}}\left[\left(\mathfrak{w}_{1}+T_{1} \mathfrak{w}_{2}\right) T_{1}+T_{2}^{2} \mathfrak{w}_{2}\right]+U_{1} \mathfrak{w}_{1}+h^{2} & =0 .
\end{aligned}
$$


Then the anomaly cancellation condition of eq. (2.14) can be rewritten as

$$
Q_{i} Q^{i}=\frac{2 U_{2}}{T_{2}}|\mathfrak{w}|^{2}+q_{n} q^{n},
$$

reproducing the integrated Bianchi identity (2.6). One notices that the contribution from the torus fibration is of the same sign as the Fermi multiplets anomaly, which will fit neatly with the perturbative dualities studied in the next sections. As was explained in [24], the integrated Bianchi identity, which is a measure of the five-brane charge, is indeed a natural T-duality invariant for this class of solutions, as far as dualities along the fivebrane worldvolume coordinates, that include the two-torus, are concerned.

Integrating out classically the massive gauge fields, one finds as expected a $T^{2} \hookrightarrow \mathcal{C}_{6} \rightarrow$ $K 3$ geometry with non-zero torsion, with $\mathcal{N}=2$ spacetime supersymmetry [3]. Naturally, quantum corrections need to be taken into account in order to get the superconformal non-linear sigma-model at the infrared fixed point.

For future reference let us simplify the Lagrangian associated with (2.12), using the conditions (2.15) and eq. (A.31):

$$
L_{\text {fib }}=L_{\text {free }}+\frac{U_{2}}{T_{2}} \int \mathrm{d}^{2} \theta^{+}\left[|\mathfrak{m}|^{2} \mathcal{A} \mathcal{V}-\frac{i}{2} \mathcal{A}\left(\operatorname{Re}(\mathfrak{m}) \partial_{-}\left(\Omega_{1}-\bar{\Omega}_{1}\right)+\operatorname{Re}\left(T^{\star} \mathfrak{m}\right) \partial_{-}\left(\Omega_{2}-\bar{\Omega}_{2}\right)\right)\right] .
$$

This expression makes clear that the coupling between the gauge superfields and the chiral superfields is only left-moving, since only terms in $\partial_{-} \omega_{\ell}$ appear in the $\theta^{+} \bar{\theta}^{+}$component.

Adding to the base GLSM Lagrangian (2.7) the fiber Lagrangian (2.17) defines a consistent quantum theory; it is generically possible to arrange the global charges of the fields to get non-anomalous chiral $\mathrm{U}(1)_{L}$ and $\mathrm{U}(1)_{R}[6]$, which are necessary conditions to get a superconformal fixed point with an appropriate GSO projection.

\subsection{Moduli quantization}

The relations (2.15) can be seen as quantization conditions for the torus moduli $U$ and $T$. Defining the complex charge

$$
\mathfrak{w}=\mathfrak{w}_{1}+T \mathfrak{w}_{2},
$$

one finds the constraints:

$$
\begin{array}{r}
\frac{U_{2}}{T_{2}} \operatorname{Re}(\mathfrak{w})-U_{1} \mathfrak{w}_{2} \in \mathbb{Z}, \\
\frac{U_{2}}{T_{2}} \operatorname{Re}\left(T^{\star} \mathfrak{w}\right)+U_{1} \mathfrak{w}_{1} \in \mathbb{Z} .
\end{array}
$$

Similar conditions arise in supergravity by considering flux quantization, see e.g. [37], and were discussed at the GLSM level in [3]. However the effect of the constant $B$-field (i.e. of $U_{1} \neq 0$ ) was not taken into account there. As we shall see below, this $B$-field - that arises naturally by T-dualizing a non-orthogonal torus fibration - plays an important role in describing the moduli space of allowed torus fibration over K3. 
Moduli space of torus fibers. In torsional GLSMs with a single U(1) gauge group, the quantization conditions (2.19) reduce the complex two-dimensional moduli space parametrized by $(U, T)$ to a one-dimensional subspace, which is described as follows. Under an arbitrary infinitesimal complex structure deformation $\delta T$, the Kähler structure has to change as well, in order to preserve the conditions (2.19). The curve defined by the intersection of the two hypersurfaces corresponding to (2.15) is locally given by

$$
\delta U=-\frac{U_{2}}{T_{2}} \frac{\mathfrak{w}}{\overline{\mathfrak{w}}} \times \overline{\delta T}
$$

More generic models are just as easy to consider. One starts with a K3 GLSM with a $\mathrm{U}(1)^{r}$ gauge symmetry, and a complexified two-torus fiber with a set of complex charges $\mathfrak{w}^{n}, n=1, \ldots, r$. For each of these charges one finds quantization conditions of the form (2.19). Hence one gets as many relations (2.20) between the complex structure and Kähler structure deformations of the two-torus. If the complex charges $\mathfrak{w}^{n}$ are not parallel to each other, the moduli are completely frozen to discrete values. We shall however see below that, even with a U(1) gauge group, this turns out to be the case.

Further constraints from dualities. Let us consider the elliptic curve $E_{T}=\mathbb{C} /(\mathbb{Z}+T \mathbb{Z})$ associated with the complex structure of the two-torus fiber. Anticipating on the results of section 3, by imposing invariance of the theory under T-duality we obtain that $\mathfrak{w} \bar{U}$ should belong to the same charge lattice as $\mathfrak{w}$, for a generic choice of complex topological charge $\mathfrak{w}$; this constraint is actually rather strong.

Setting apart the trivial cases with $\mathfrak{w} \bar{U} \in \mathbb{Z}$, this condition means that the elliptic curve $E_{T}$ should admit a non-trivial endomorphism:

$$
\begin{aligned}
E_{T} & \rightarrow E_{T} \\
z & \mapsto \mathfrak{w} \bar{U} z
\end{aligned}
$$

This property, known as complex multiplication, it only shared by elliptic curves whose complex structure $T$ is valued in an imaginary quadratic number field $\mathbb{Q}(\sqrt{D})$, namely

$$
T \in \mathbb{Q}+\sqrt{D} \mathbb{Q} \quad \text { with } \quad D=b^{2}-4 a c<0, \quad a, b, c, d \in \mathbb{Z}
$$

For a nice presentation of these topics from a string theory perspective, see [38].

To be more explicit, the condition for $\phi$ to be an automorphism of $E_{T}$ can be solved with the sufficient conditions

$$
\left\{\begin{array}{l}
\exists\left(m_{1}, n_{1}\right) \in \mathbb{Z}^{2}, \mathfrak{w} \bar{U}=m_{1}+T n_{1} \\
\exists\left(m_{2}, n_{2}\right) \in \mathbb{Z}^{2}, \mathfrak{w} \bar{U} T=m_{2}+T n_{2}
\end{array} .\right.
$$

These relations lead to a second-order equation for the complex structure $T$ with integer coefficients, hence $T$ belongs to some $\mathbb{Q}(\sqrt{D})$. Furthermore, eq. (2.23) implies that both $U$ 
and $T$ are valued in the same imaginary quadratic field. Whenever this is the case, the quantization conditions (2.19) are easily solved since they now involve only rational numbers. ${ }^{7}$

Interestingly, a two-dimensional conformal field theory with a two-torus target space whose complex structure $T$ and Kähler structure $U$ are valued in the same imaginary quadratic number field $\mathbb{Q}(\sqrt{D})$ is a rational conformal field theory [39], i.e. with an extended chiral algebra with respect to which the number of primary fields is of finite number. ${ }^{8}$ This peculiar quantization of the moduli fits neatly with known facts about string duals of the heterotic solutions under consideration, as we shall see below.

Let us consider a type IIB orientifold $K 3 \times T^{2} / \mathbb{Z}_{2}$ with flux [14], where the orientifold action is defined as $\mathbb{Z}_{2}=\Omega(-)^{F_{L}} \mathcal{I}$ with $\mathcal{I}$ the inversion along the torus; we take a square torus for simplicity. The type IIB string-frame metric for the compact space is of the form (we use thereafter tilded variables to denote type IIB quantities)

$$
\mathrm{d} \tilde{s}^{2}=\Delta\left[\tilde{R}_{1}^{2}\left(\mathrm{~d} \tilde{x}^{1}\right)^{2}+\tilde{R}_{2}^{2}\left(\mathrm{~d} \tilde{x}^{2}\right)^{2}+\mathrm{d} s^{2}(K 3)\right],
$$

where the overall warp factor $\Delta$ is a function of the K3 coordinates. As explained in $[38,41], \mathcal{N}=2$-preserving fluxes fix neither the complex structure of K3 nor the Kähler structure of the $T^{2}$ completely, but constrains the complex structure of the torus $\tilde{T}$ and the axio-dilaton $\tilde{\Phi}=a+i / \tilde{g}_{s}$, which is constant in this type IIB background, to be valued in the same imaginary quadratic number field.

Mapping this type IIB orientifold compactification first to type I (by using the $U \rightarrow$ $-1 / U$ and $T \rightarrow-1 / T$ elements of the toroidal T-duality group along the two-torus), then to a $\operatorname{Spin}(32) / \mathbb{Z}_{2}$ heterotic solution by using S-duality, leads to the string-frame metric

$$
\mathrm{d} s^{2}=\frac{1}{\tilde{g}_{s}} \frac{\tilde{R}_{2}}{\tilde{R}_{1}}\left[\left(\mathrm{~d} x^{1}+\pi^{\star} \mathfrak{w}_{1, \ell} \alpha^{\ell}\right)^{2}+\left(\frac{\tilde{R}_{1}}{\tilde{R}_{2}}\right)^{2}\left(\mathrm{~d} x^{2}+\pi^{\star} \mathfrak{w}_{2, \ell} \alpha^{\ell}\right)^{2}\right]+\frac{\tilde{R}_{1} \tilde{R}_{2}}{\tilde{g}_{s}} \Delta^{2} \mathrm{~d} s^{2}(K 3) .
$$

The torus is now fibered over K3, as follows from T-dualizing the NSNS two-form of the type IIB solution.

This heterotic background is of the same type as those given by eq. (2.1), upon identifying the warp factor as $e^{2 A}=\frac{\tilde{R}_{1} \tilde{R}_{2}}{\tilde{g}_{s}} \Delta^{2}$ and the torus moduli as $T_{2}=\tilde{R}_{1} / \tilde{R}_{2}=1 / \tilde{T}_{2}$ and $U_{2}=1 / \tilde{g}_{2}$. More generally, the chain of dualities relates the type IIB and heterotic moduli as follows (up to a $\operatorname{PSL}(2, \mathbb{Z})_{T}$ transformation):

$$
T_{\mathrm{HET}}=T_{\mathrm{IIB}}, \quad U_{\mathrm{HET}}=\Phi_{\mathrm{IIB}},
$$

\footnotetext{
${ }^{7}$ Likewise, under T-duality along $x^{1}$, which exchanges the complex and Kähler structures, one has the map $\mathfrak{w} \mapsto \frac{U_{2}}{T_{2}} \mathfrak{w}$. Therefore, as the dual charge has to belong to the lattice $\mathbb{Z}+U \mathbb{Z}$, one has the constraint

$$
\exists\left(r_{1}, r_{2}\right) \in \mathbb{Z}^{2}, \quad \frac{U_{2}}{T_{2}}\left(\mathfrak{w}_{1}+T \mathfrak{w}_{2}\right)=r_{1}+U r_{2},
$$

which is automatically satisfied using the quantization condition (2.19a).

${ }^{8}$ When the base is an Eguchi-Hanson space, in which case a worldsheet CFT is known, this requirement is easy to understand [40]. The existence of chiral generators of an extended algebra is actually needed in order to define the super-Liouville potential that appears in these constructions.
} 
hence mapping the quantization conditions for the axio-dilaton and torus complex structure found in type IIB to the quantization conditions for both torus moduli in heterotic, that we have found above.

To take another point of view, heterotic strings on $K 3 \times T^{2}$, with the two-torus at a rational conformal field theory point (i.e. with $T, U \in \mathbb{Q}(\sqrt{D})$ as we have seen) is dual to $\mathcal{F}$-theory on $K_{3} \times K 3^{\prime}$, where the $K 3^{\prime}$ surface is of a special type, called attractive by Greg Moore [38], having its Picard group of maximal dimension. We refer to this reference for more details about their properties. Adding flux to these $\mathcal{F}$-theory compactifications allows one to get the torsional solutions of interest in heterotic. Conditions imposed by G-flux have been analysed in the $\mathcal{M}$-theory dual [14], and precisely single out attractive K3 surfaces.

\section{Torus self-duality}

In this section we study the action of perturbative dualities along the two-torus fiber. In order to derive these dualities $\grave{a}$ la Buscher from the worldsheet theory, we add an extra U(1) gauge symmetry (denoted with a hat thereafter) to the torsional GLSM, corresponding to the pair of superfields $(\hat{\mathcal{A}}, \hat{\mathcal{V}})$, and gauge the imaginary shift-symmetry of the superfields $\Omega_{\ell}$.

\subsection{Warm-up}

For starters, let us consider a free chiral superfield $\Phi$ whose bottom component parametrizes a cylinder $\mathbb{C}^{\star} \simeq \mathbb{R} \times S^{1}$, with $\phi \sim \phi+2 i \pi$. Gauging the (compact) shift symmetry of the imaginary part of $\phi$, we consider the Lagrangian superspace density:

$$
L=-\frac{i R^{2}}{8}(\Phi+\bar{\Phi}+2 \hat{\mathcal{A}})\left(\partial_{-}(\Phi-\bar{\Phi})+2 i \hat{\mathcal{V}}\right)+\frac{1}{4}\left(\hat{\mathcal{V}}(\Delta+\bar{\Delta})+i \hat{\mathcal{A}} \partial_{-}(\Delta-\bar{\Delta})\right)
$$

Integrating out $\Delta$ first yields the equation

$$
0=\bar{D}_{+}\left(\partial_{-} \hat{\mathcal{A}}+i \hat{\mathcal{V}}\right)=\hat{\Upsilon}
$$

hence giving back the original action.

In order to obtain the dual description, one integrates out first the gauge fields instead. Fixing the the gauge with $\Phi=0$, one gets the equations of motion

$$
R^{2} \hat{\mathcal{V}}=-\frac{i}{2} \partial_{-}(\Delta-\bar{\Delta}), \quad R^{2} \hat{\mathcal{A}}=-\frac{1}{2}(\Delta+\bar{\Delta})
$$

Therefore the dual Lagrangian density reads

$$
\tilde{L}=-\frac{i}{8 R^{2}}(\Delta+\bar{\Delta}) \partial_{-}(\Delta-\bar{\Delta})
$$

which is the expected $R \leftrightarrow \frac{1}{R}$ toroidal T-duality as far as $\operatorname{Im} \phi$ is concerned. 


\section{Minimally vs axially coupled chirals}

It is also possible to dualize a chiral superfield $\Phi$ whose shift-symmetry was already gauged, associated with a pair of gauge superfields $(\mathcal{A}, \mathcal{V})$, and with shift-charge $q$. One considers the following Lagrangian density in superspace (omitting the kinetic term of $\Upsilon$ that plays no role):

$$
\begin{aligned}
L= & -\frac{i R^{2}}{8}(\Phi+\bar{\Phi}+2 q \mathcal{A}+2 \hat{\mathcal{A}})\left(\partial_{-}(\Phi-\bar{\Phi})+2 i q \mathcal{V}+2 i \hat{\mathcal{V}}\right) \\
& +\frac{1}{4}\left(\hat{\mathcal{V}}(\Delta+\bar{\Delta})+i \hat{\mathcal{A}} \partial_{-}(\Delta-\bar{\Delta})\right) .
\end{aligned}
$$

Integrating out the gauge superfields $\hat{\mathcal{A}}$ and $\hat{\mathcal{V}}$ in the gauge $\Phi=0$ gives the dual model, corresponding to a neutral chiral superfield axially coupled to the gauge superfields $(\mathcal{A}, \mathcal{V})$ through a FI-like term:

$$
\tilde{L}=-\frac{i}{8 R^{2}}(\Delta+\bar{\Delta}) \partial_{-}(\Delta-\bar{\Delta})-\frac{q}{4}\left(\mathcal{V}(\Delta+\bar{\Delta})+i \mathcal{A} \partial_{-}(\Delta-\bar{\Delta})\right) .
$$

In the opposite situation, one starts with an axially coupled neutral chiral superfield with axial coupling $h \in \mathbb{Z}$ :

$$
\frac{h}{4}\left(\mathcal{V}(\Phi+\bar{\Phi})+i \mathcal{A} \partial_{-}(\Phi-\bar{\Phi})\right)
$$

One minimally couples this superfield to the hatted gauge superfields as in eq. (3.1). However, the axial coupling (3.7) is not invariant under the newly introduced hatted gauge symmetry. Hence the latter should be corrected to

$$
L_{A}=\frac{h}{4}\left[\mathcal{V}(\Phi+\bar{\Phi}+2 \hat{\mathcal{A}})+i \mathcal{A}\left(\partial_{-}(\Phi-\bar{\Phi})+2 i \hat{\mathcal{V}}\right)\right]
$$

Notice that, under an unhatted gauge transformation, this axial coupling shifts by the term

$$
\delta_{\Xi} L_{A}=\frac{i h}{4}\left(\Xi\left(\hat{\mathcal{V}}-i \partial_{-} \hat{\mathcal{A}}\right)+\Xi\left(\hat{\mathcal{V}}+i \partial_{-} \hat{\mathcal{A}}\right)\right)
$$

which looks like a gauge anomaly for the hatted $\mathrm{U}(1)$ supergauge symmetry but for a gauge transformation of the original $\mathrm{U}(1)$. In order to compensate for this classical gauge non-invariance, we need to assign a shift charge $-h$ w.r.t. the unhatted gauge symmetry to the chiral superfield $\Delta$. Then the gauge variation of the coupling

$$
L_{\Delta}=\frac{1}{4}\left(\hat{\mathcal{V}}(\Delta+\bar{\Delta})+i \hat{\mathcal{A}} \partial_{-}(\Delta-\bar{\Delta})\right)
$$

precisely cancels (3.9). Integrating out the hatted gauge fields in the gauge $\Phi=0$ gives the dual Lagrangian density

$$
\tilde{L}=-\frac{i}{8 R^{2}}(\Delta+\bar{\Delta}-2 h \mathcal{A})\left(\partial_{-}(\Delta-\bar{\Delta})-2 i h \mathcal{V}\right)
$$

which is indeed a minimally coupled chiral field of shift charge $\tilde{q}=-h$. 


\subsection{Dualizing the fibered torus}

We consider now the model of interest, namely a torsional GLSM for a $T^{2} \hookrightarrow \mathcal{C}_{6} \rightarrow K 3$ compactification. Our aim is to show that this set of compactifications is closed under $O(2,2, \mathbb{Z})$ perturbative dualities along the torus fiber. We will derive the transformation rules for the charge doublet $\left(\mathfrak{w}_{1}, \mathfrak{w}_{2}\right)$ under such transformations. Dualities belonging to the larger $O(2,2+N, \mathbb{Z})$ heterotic duality group will be considered in the next section.

The K3 GLSM plays no role in the duality, hence we write below only the terms in the Lagrangian density associated with the torus fiber for generic moduli $U$ and $T$, see eq. (2.12). In contrast with the previous examples, the chiral superfields are coupled axially and minimally at the same time. Our aim is to find a set of duality transformations generating the full $\operatorname{PSL}(2, \mathbb{Z}))_{T} \times \operatorname{PSL}(2, \mathbb{Z})_{U} \times \mathbb{Z}_{2} \times \mathbb{Z}_{2}$ perturbative duality group.

Let us dualize the chiral superfield $\Omega_{1}$ first, coupling also $\Omega_{2}$ axially to the hatted gauge superfields, with a parameter $s \in \mathbb{Z}$. One starts then with the following superspace Lagrangian density:

$$
\begin{aligned}
L_{s}=-\frac{i U_{2}}{8 T_{2}}\left(\Omega_{1}+\bar{\Omega}_{1}+T_{1}\left(\Omega_{2}+\bar{\Omega}_{2}\right)+2\left(\mathfrak{w}_{1}+T_{1} \mathfrak{w}_{2}\right) \mathcal{A}+2 \hat{\mathcal{A}}\right) \times \\
\times\left(\partial_{-}\left(\Omega_{1}-\bar{\Omega}_{1}+T_{1}\left(\Omega_{2}-\bar{\Omega}_{2}\right)\right)+2 i\left(\mathfrak{w}_{1}+T_{1} \mathfrak{w}_{2}\right) \mathcal{V}+2 i \hat{\mathcal{V}}\right) \\
\quad-\frac{i}{8} U_{2} T_{2}\left(\Omega_{2}+\bar{\Omega}_{2}+2 \mathfrak{w}_{2} \mathcal{A}\right)\left(\partial_{-}\left(\Omega_{2}-\bar{\Omega}_{2}\right)+2 i \mathfrak{w}_{2} \mathcal{V}\right) \\
+\frac{i}{8} U_{1}\left[\left(\Omega_{1}+\bar{\Omega}_{1}+2 \mathfrak{w}_{1} \mathcal{A}+2 \hat{\mathcal{A}}\right)\left(\partial_{-}\left(\Omega_{2}-\bar{\Omega}_{2}\right)+2 i \mathfrak{w}_{2} \mathcal{V}\right)-\right. \\
\left.\quad-\left(\Omega_{2}+\bar{\Omega}_{2}+2 \mathfrak{w}_{2} \mathcal{A}\right)\left(\partial_{-}\left(\Omega_{1}-\bar{\Omega}_{1}\right)+2 i \mathfrak{w}_{1} \mathcal{V}+2 i \hat{\mathcal{V}}\right)\right] \\
+\frac{h_{1}}{4}\left[\mathcal{V}\left(\Omega_{1}+\bar{\Omega}_{1}+2 \hat{\mathcal{A}}\right)+i \mathcal{A}\left(\partial_{-}\left(\Omega_{1}-\bar{\Omega}_{1}\right)+2 i \hat{\mathcal{V}}\right)\right]+\frac{h_{2}}{4}\left[\mathcal{V}\left(\Omega_{2}+\bar{\Omega}_{2}\right)+i \mathcal{A} \partial_{-}\left(\Omega_{2}-\bar{\Omega}_{2}\right)\right] \\
+\frac{1}{4}\left[\hat{\mathcal{V}}(\Theta+\bar{\Theta})+i \hat{\mathcal{A}} \partial_{-}(\Theta-\bar{\Theta})\right]+\frac{s}{4}\left[\hat{\mathcal{V}}\left(\Omega_{2}+\bar{\Omega}_{2}\right)+i \hat{\mathcal{A}} \partial_{-}\left(\Omega_{2}-\bar{\Omega}_{2}\right)\right] . \quad(3.11)
\end{aligned}
$$

Neither $\Theta$ nor $\Omega_{2}$ is charged under the hatted gauge symmetry hence gauge invariance w.r.t. the latter is automatic.

Gauge-invariance w.r.t. the unhatted gauge symmetry, corresponding to the superfields $\mathcal{A}$ and $\mathcal{V}$, should be examined more carefully. Assigning a shift-charge $\mathfrak{w}_{\theta}$ to the chiral superfield $\Theta$, under a super-gauge variation, the Lagrangian density (3.11) is classically shifted by the term (after integration by parts)

$$
\delta_{\Xi} L_{s}=\frac{h^{\ell} \mathfrak{w}_{\ell}}{4} \int \mathrm{d} \theta^{+} \Xi \Upsilon+\frac{1}{4}\left(h_{1}+\mathfrak{w}_{\theta}+s \mathfrak{w}_{2}\right) \int \mathrm{d} \theta^{+} \Xi \hat{\Upsilon}+h . c .
$$

The first term is, as above, responsible for canceling the gauge anomaly coming from the base GLSM, see eq. (2.14). The second term should vanish, thereby fixing the value of the $\Theta$ shift-charge to (see the discussion below (3.9)):

$$
\mathfrak{w}_{\theta}=-h_{1}-s \mathfrak{w}_{2}=\frac{U_{2}}{T_{2}}\left(\mathfrak{w}_{1}+T_{1} \mathfrak{w}_{2}\right)-\left(U_{1}+s\right) \mathfrak{w}_{2}
$$

where we used the relation $(2.15)$. 
After being reassured that gauge-invariance is satisfied, one can simplify the bulky expression (3.11) by using one more time eq. (2.15) and by fixing the gauge $\Omega_{1}=0$ :

$$
\begin{aligned}
& L_{s}=-\frac{i}{8} \frac{U_{2}}{T_{2}}|T|^{2}\left(\Omega_{2}+\bar{\Omega}_{2}\right) \partial_{-}\left(\Omega_{2}-\bar{\Omega}_{2}\right)+\frac{U_{2}}{2 T_{2}}|\mathfrak{m}|^{2} \mathcal{A} \mathcal{V}+\frac{i}{2} \mathcal{A}\left(h_{2}+U_{1} \mathfrak{w}_{1}\right) \partial_{-}\left(\Omega_{2}-\bar{\Omega}_{2}\right) \\
& +\frac{U_{2}}{2 T_{2}} \hat{\mathcal{A}} \hat{\mathcal{V}}-\left(-U_{1} \mathfrak{w}_{2}+h_{1}\right) \mathcal{A} \hat{\mathcal{V}}+\frac{1}{4}\left[\hat{\mathcal{V}}\left(\Theta+\bar{\Theta}+U_{1}\left(\Omega_{2}+\bar{\Omega}_{2}\right)\right)+i \hat{\mathcal{A}} \partial_{-}\left(\Theta-\bar{\Theta}+U_{1}\left(\Omega_{2}-\bar{\Omega}_{2}\right)\right)\right] \\
& +\frac{1}{4} \frac{U_{2} T_{1}}{T_{2}}\left[\hat{\mathcal{V}}\left(\Omega_{2}+\bar{\Omega}_{2}\right)-i \hat{\mathcal{A}} \partial_{-}\left(\Omega_{2}-\bar{\Omega}_{2}\right)\right]+\frac{s}{4}\left[\hat{\mathcal{V}}\left(\Omega_{2}+\bar{\Omega}_{2}\right)+i \hat{\mathcal{A}} \partial_{-}\left(\Omega_{2}-\bar{\Omega}_{2}\right)\right] .
\end{aligned}
$$

Now integrating out the hatted gauge superfields gives

$$
\begin{aligned}
& \hat{\mathcal{V}}=-\frac{i}{2} \frac{T_{2}}{U_{2}} \partial_{-}\left(\Theta-\bar{\Theta}+\left(U_{1}+s\right)\left(\Omega_{2}-\bar{\Omega}_{2}\right)\right)+\frac{i}{2} T_{1} \partial_{-}\left(\Omega_{2}-\bar{\Omega}_{2}\right), \\
& \hat{\mathcal{A}}=-\frac{1}{2} \frac{T_{2}}{U_{2}}\left(\Theta+\bar{\Theta}+\left(U_{1}+s\right)\left(\Omega_{2}+\bar{\Omega}_{2}\right)\right)+\frac{T_{2}}{U_{2}}\left(-2 U_{1} \mathfrak{w}_{2}+2 h_{1}\right) \mathcal{A}-\frac{1}{2} T_{1}\left(\Omega_{2}+\bar{\Omega}_{2}\right),
\end{aligned}
$$

leading to the dual Lagrangian density:

$$
\begin{aligned}
& \tilde{L}=-\frac{i}{8} \frac{T_{2}}{U_{2}}\left(\Theta+\bar{\Theta}+\left(U_{1}+s\right)\left(\Omega_{2}+\bar{\Omega}_{2}\right)\right) \partial_{-}\left(\Theta-\bar{\Theta}+\left(U_{1}+s\right)\left(\Omega_{2}-\bar{\Omega}_{2}\right)\right) \\
& -\frac{i}{8} U_{2} T_{2}\left(\Omega_{2}+\bar{\Omega}_{2}\right) \partial_{-}\left(\Omega_{2}-\bar{\Omega}_{2}\right)-\frac{i}{8} T_{1}\left(\left(\Omega_{2}+\bar{\Omega}_{2}\right) \partial_{-}(\Theta-\bar{\Theta})-(\Theta+\bar{\Theta}) \partial_{-}\left(\Omega_{2}-\bar{\Omega}_{2}\right)\right) \\
& +\frac{U_{2}}{2 T_{2}}|\mathfrak{w}|^{2} \mathcal{A} \mathcal{V}-\frac{i}{2}\left[U_{2} T_{2} \mathfrak{w}_{2}+\left(\mathfrak{w}_{1}+T_{1} \mathfrak{w}_{2}\right)\left(U_{1}+s\right)\right] \mathcal{A} \partial_{-}\left(\Omega_{2}-\bar{\Omega}_{2}\right)-\frac{i}{2}\left(\mathfrak{w}_{1}+T_{1} \mathfrak{w}_{2}\right) \mathcal{A} \partial_{-}(\Theta-\bar{\Theta}) .
\end{aligned}
$$

We read then the duality transformations of the torus moduli from the first two lines of eq. (3.16):

$$
\mathcal{G}_{s}:\left\{\begin{array}{l}
U \mapsto \tilde{U}=T \\
T \mapsto \tilde{T}=U+s
\end{array} .\right.
$$

Given that the dual Lagrangian is of the same form as the original one, we define the dual complex topological charge with the help of the dual complex structure:

$$
\tilde{\mathfrak{w}}=\tilde{\mathfrak{w}}_{1}+\tilde{T} \tilde{\mathfrak{w}}_{2}=\tilde{\mathfrak{w}}_{1}+(U+s) \tilde{\mathfrak{w}}_{2}
$$

Comparing with the generic model, given by eq. (2.12), we find the following duality map between the charges specifying the principal bundle:

$$
\begin{aligned}
\operatorname{Re}(\tilde{\mathfrak{w}}) & =\frac{U_{2}}{T_{2}} \operatorname{Re}(\mathfrak{w}), \\
\operatorname{Re}((\bar{U}+s) \tilde{\mathfrak{w}}) & =\frac{U_{2}}{T_{2}} \operatorname{Re}((\bar{U}+s) \mathfrak{w}) .
\end{aligned}
$$

We consider now dualizing the chiral superfield $\Omega_{2}$ instead. One starts with the superspace Lagrangian density

$$
\begin{aligned}
& L=-\frac{i U_{2}}{8 T_{2}}(\left.\Omega_{1}+\bar{\Omega}_{1}+T_{1}\left(\Omega_{2}+\bar{\Omega}_{2}\right)+2\left(\mathfrak{w}_{1}+T_{1} \mathfrak{w}_{2}\right) \mathcal{A}+2 T_{1} \hat{\mathcal{A}}\right) \times \\
& \times\left(\partial_{-}\left(\Omega_{1}-\bar{\Omega}_{1}+T_{1}\left(\Omega_{2}-\bar{\Omega}_{2}\right)\right)+2 i\left(\mathfrak{w}_{1}+T_{1} \mathfrak{w}_{2}\right) \mathcal{V}+2 i T_{1} \hat{\mathcal{V}}\right)
\end{aligned}
$$




$$
\begin{gathered}
-\frac{i}{8} U_{2} T_{2}\left(\Omega_{2}+\bar{\Omega}_{2}+2 \mathfrak{w}_{2} \mathcal{A}+2 \hat{\mathcal{A}}\right)\left(\partial_{-}\left(\Omega_{2}-\bar{\Omega}_{2}\right)+2 i \mathfrak{w}_{2} \mathcal{V}+2 i \hat{\mathcal{V}}\right) \\
+\frac{i}{8} U_{1}\left[\left(\Omega_{1}+\bar{\Omega}_{1}+2 \mathfrak{w}_{1} \mathcal{A}\right)\left(\partial_{-}\left(\Omega_{2}-\bar{\Omega}_{2}\right)+2 i \mathfrak{w}_{2} \mathcal{V}+2 i \hat{\mathcal{V}}\right)-\right. \\
\left.-\left(\Omega_{2}+\bar{\Omega}_{2}+2 \mathfrak{w}_{2} \mathcal{A}+2 \hat{\mathcal{A}}\right)\left(\partial_{-}\left(\Omega_{1}-\bar{\Omega}_{1}\right)+2 i \mathfrak{w}_{1} \mathcal{V}\right)\right] \\
+\frac{h_{1}}{4}\left[\mathcal{V}\left(\Omega_{1}+\bar{\Omega}_{1}\right)+i \mathcal{A} \partial_{-}\left(\Omega_{1}-\bar{\Omega}_{1}\right)\right] \\
+\frac{h_{2}}{4}\left[\mathcal{V}\left(\Omega_{2}+\bar{\Omega}_{2}+2 \hat{\mathcal{A}}\right)+i \mathcal{A}\left(\partial_{-}\left(\Omega_{2}-\bar{\Omega}_{2}\right)+2 i \hat{\mathcal{V}}\right)\right]+\frac{1}{4}\left[\hat{\mathcal{V}}(\Theta+\bar{\Theta})+i \hat{\mathcal{A}} \partial_{-}(\Theta-\bar{\Theta})\right] .
\end{gathered}
$$

Integrating out the hatted gauge fields and taking the gauge $\Omega_{2}=0$, one is left to the dual Lagrangian

$$
\begin{aligned}
\tilde{L}=-\frac{i}{8} \frac{U_{2} T_{2}}{|T|^{2}}\left(\Omega_{1}+\bar{\Omega}_{1}\right) \partial_{-}\left(\Omega_{1}-\bar{\Omega}_{1}\right) \\
\quad-\frac{i}{8} \frac{T_{2}}{U_{2}|T|^{2}}\left(\Theta+\bar{\Theta}-U_{1}\left(\Omega_{1}+\bar{\Omega}_{1}\right)\right) \partial_{-}\left(\Theta-\bar{\Theta}-U_{1}\left(\Omega_{1}-\bar{\Omega}_{1}\right)\right) \\
\quad+\frac{i}{8} \frac{T_{1}}{|T|^{2}}\left[(\Theta+\bar{\Theta}) \partial_{-}\left(\Omega_{1}-\bar{\Omega}_{1}\right)-\left(\Omega_{1}+\bar{\Omega}_{1}\right) \partial_{-}(\Theta-\bar{\Theta})\right] \\
+\frac{U_{2}}{2 T_{2}}|\mathfrak{w}|^{2} \mathcal{A} \mathcal{V}-\frac{i}{2} \frac{\operatorname{Re}(\bar{T} \mathfrak{w})}{|T|^{2}} \mathcal{A} \partial_{-}(\Theta-\bar{\Theta})+\frac{i}{2} \frac{\operatorname{Re}(\overline{T U} \mathfrak{w})}{|T|^{2}} \mathcal{A} \partial_{-}\left(\Omega_{1}-\bar{\Omega}_{1}\right) .
\end{aligned}
$$

Hence the torus moduli transform as follows:

$$
\mathcal{H}:\left\{\begin{array}{l}
U \mapsto \tilde{U}=\frac{1}{\bar{T}} \\
T \mapsto \tilde{T}=-\bar{U}
\end{array} .\right.
$$

Accordingly, defining now $\tilde{\mathfrak{w}}=\tilde{\mathfrak{w}}_{1}-\bar{U} \tilde{\mathfrak{w}}_{2}$, one has

$$
\begin{aligned}
\operatorname{Re}(\tilde{\mathfrak{w}}) & =\frac{U_{2}}{T_{2}} \operatorname{Re}(\bar{T} \mathfrak{w}), \\
\operatorname{Re}(U \tilde{\mathfrak{w}}) & =\frac{U_{2}}{T_{2}} \operatorname{Re}(\overline{T U} \mathfrak{w}) .
\end{aligned}
$$

Had we had chosen instead the axial coupling of $\Theta$ with an opposite sign, one would end up with another dual Lagrangian density

$$
\begin{aligned}
\tilde{L}=-\frac{i}{8} \frac{U_{2} T_{2}}{|T|^{2}}\left(\Omega_{1}+\bar{\Omega}_{1}\right) \partial_{-}\left(\Omega_{1}-\bar{\Omega}_{1}\right) \\
\quad-\frac{i}{8} \frac{T_{2}}{U_{2}|T|^{2}}\left(\Theta+\bar{\Theta}+U_{1}\left(\Omega_{1}+\bar{\Omega}_{1}\right)\right) \partial_{-}\left(\Theta-\bar{\Theta}+U_{1}\left(\Omega_{1}-\bar{\Omega}_{1}\right)\right) \\
\quad-\frac{i}{8} \frac{T_{1}}{|T|^{2}}\left[(\Theta+\bar{\Theta}) \partial_{-}\left(\Omega_{1}-\bar{\Omega}_{1}\right)-\left(\Omega_{1}+\bar{\Omega}_{1}\right) \partial_{-}(\Theta-\bar{\Theta})\right] \\
\left.+\frac{U_{2}}{2 T_{2}}|\mathfrak{w}|^{2} \mathcal{A} \mathcal{V}+\frac{i}{2} \frac{\operatorname{Re}(\bar{T} \mathfrak{w})}{|T|^{2}} \mathcal{A} \partial_{-}(\Theta-\bar{\Theta})\right)+\frac{i}{2} \frac{\operatorname{Re}(\overline{T U} \mathfrak{w})}{|T|^{2}} \mathcal{A} \partial_{-}\left(\Omega_{1}-\bar{\Omega}_{1}\right),
\end{aligned}
$$

corresponding to the transformations

$$
\mathcal{I}:\left\{\begin{array}{l}
U \mapsto \tilde{U}=-\frac{1}{T} \\
T \mapsto \tilde{T}=U
\end{array} .\right.
$$


As before, defining $\tilde{\mathfrak{w}}=\tilde{\mathfrak{w}}_{1}+U \tilde{\mathfrak{w}}_{2}$ leads to the map

$$
\begin{aligned}
\operatorname{Re}(\tilde{\mathfrak{w}}) & =-\frac{U_{2}}{T_{2}} \operatorname{Re}(\bar{T} \mathfrak{w}), \\
\operatorname{Re}(\bar{U} \tilde{\mathfrak{w}}) & =-\frac{U_{2}}{T_{2}} \operatorname{Re}(\overline{T U} \mathfrak{w})
\end{aligned}
$$

\subsection{Perturbative duality group}

The set of duality transformations that were derived above by expressing them as quotients allows one to generate the full $O(2,2, \mathbb{Z})$ perturbative duality group along the two-torus fiber.

First, let us discuss the $\operatorname{PSL}(2, \mathbb{Z})_{T}$ factor, acting on the complex structure. The $\mathcal{T}_{T}$ and $\mathcal{S}_{T}$ generators are respectively given in terms of the previously defined dualities as $\mathcal{G}_{1} \mathcal{G}_{0}$ and $\mathcal{G}_{0} \mathcal{I}$. The corresponding duality transformations for the complex charge $\mathfrak{w}$ are then given by

$$
\mathcal{T}_{T}: \mathfrak{w} \mapsto \mathfrak{w}, \quad \mathcal{S}_{T}: \mathfrak{w} \mapsto-\frac{1}{T} \mathfrak{w} .
$$

In other words, $\left(\mathfrak{w}_{1}, \mathfrak{w}_{2}\right)$ transforms as a doublet of $\operatorname{PSL}(2, \mathbb{Z})_{T}$. Under $T \rightarrow \frac{a T+b}{c T+d}$ one has the map

$$
\left(\begin{array}{c}
\mathfrak{w}_{1} \\
\mathfrak{w}_{2}
\end{array}\right) \mapsto\left(\begin{array}{cc}
a & b \\
c & d
\end{array}\right)\left(\begin{array}{c}
\mathfrak{w}_{1} \\
\mathfrak{w}_{2}
\end{array}\right),
$$

which is not a surprising result.

The $\operatorname{PSL}(2, \mathbb{Z})_{U}$ factor, acting on the Kähler structure, is generated by the elements $\mathcal{T}_{U}=\mathcal{G}_{0} \mathcal{G}_{1}$ and $\mathcal{S}_{U}=\mathcal{I} \mathcal{G}_{0}$. Using the previous results one finds that the complex topological charge transforms under these dualities as:

$$
\mathcal{T}_{U}: \mathfrak{w} \mapsto \mathfrak{w}, \quad \mathcal{S}_{U}: \mathfrak{w} \mapsto-\bar{U} \mathfrak{w}
$$

The behavior of $\mathfrak{w}$ under $\mathcal{S}_{U}$ makes only sense if the transformed charge $-\bar{U} \mathfrak{w}$ belongs to the same charge lattice as $\mathfrak{w}$, namely $\mathbb{Z}+T \mathbb{Z}$. Implications of this important result for moduli quantization were discussed in subsection 2.4 .

Finally, a first $\mathbb{Z}_{2}$ factor, T-duality along $x_{1}$, is directly given by $\mathcal{G}_{0}$ with the complex charge transforming as $\mathfrak{w} \rightarrow \frac{U_{2}}{T_{2}} \mathfrak{w}$. The second $\mathbb{Z}_{2}$, namely the parity transformation, corresponds to $\mathcal{I} \mathcal{G}_{0} \mathcal{H}$ and maps $\mathfrak{w} \rightarrow-\overline{\mathfrak{w}}$. This completes the perturbative duality group of the two-torus.

Hence we have proven that the whole $O(2,2, \mathbb{Z})$ perturbative duality group is an exact symmetry of the class of torsional gauged linear sigma-models under consideration, and not only a symmetry among their infrared fixed points.

One can wonder whether non-perturbative effects (a.k.a. worldsheet instantons) can alter our statements about perturbative dualities, in the same vein as in the GLSM approach to mirror symmetry [21]. In the latter case, one dualizes the phase of a chiral multiplet, hence the system reduces for all purposes to supersymmetric QED with a massless flavor. This theory admits vortex-like instanton solutions, generating in the mirror theory a superpotential. In contrast, one considers in the present case chiral 
superfields whose shift-symmetry is gauged, giving after gauge-fixing some massive Abelian gauge theory which does not admit instantons. ${ }^{9}$ This can also be understood by noticing that the target space of the shift superfields has the topology $\mathbb{C}^{\star} \sim \mathbb{R} \times S^{1}$. Hence, the previous statements about T-duality are exact; the same conclusion holds for the more generic heterotic dualities that we shall study below.

\section{Torus/bundle dualities}

In a $T^{2} \hookrightarrow \mathcal{C}_{6} \rightarrow K 3$ compactification, one expects a larger duality group, which is a subgroup of $O(2,2+16, \mathbb{Z})$. The extra duality transformations mix the torus fiber with Abelian gauge bundles. Implementing directly the relevant duality transformations at the level of the torsional GLSM seems rather difficult, since these dualities exchange classically non-gauge-invariant terms with classically gauge invariant ones suffering from quantum gauge anomalies. ${ }^{10}$

In order to overcome this difficulty, one can bosonize the free charged left-moving worldsheet fermions involved in the duality, as suggested in [5]. We shall explain below how it can be done in the present context of $(0,2)$ GLSMs.

\section{$4.1 \quad(0,2)$ bosonization of charged Fermi multiplets}

For simplicity of the presentation we consider that the non-Abelian gauge bundle $V$ over the $K 3$ base lies in the first $E_{8}$ factor. Our aim is to find under which conditions an $S^{1}$ fibration can be traded for an Abelian gauge bundle lying in the second $E_{8}$ factor. One starts then with a set of 8 Fermi multiplets $\Gamma_{n}$, having vanishing $E$ and $J$ couplings, whose charges w.r.t. the U(1) gauge symmetry of the GLSM are labeled by $f_{n}$. These Fermi multiplets are sections of line bundles over $K 3$.

The normalization of the magnetic charges in space-time can be obtained by comparing the anomaly cancellation condition in the GLSM with the Bianchi identity in supergravity. In the present GLSM approach, an Abelian gauge bundle and the torus fibration contribute generically to the gauge anomaly as $f_{n} f^{n}+\frac{2 U_{2}}{T_{2}}|\mathfrak{w}|^{2}$ (in units $\alpha^{\prime}=1$ ). In supergravity one gets the same anomaly equation (see e.g. in [40]) by considering the pullback of an Abelian bundle over $\mathrm{K} 3$ of the form $\mathcal{F}=2 \pi k_{n} \cdot T^{n} \varpi_{[2]}$, where $\varpi_{[2]} \in H^{2}(\mathcal{S}, \mathbb{Z}) \cap H^{1,1}(\mathcal{S})$ is anti-self dual, and $\left\{T_{n}, n=1, \ldots, 8\right\}$ are Cartan generators of $\mathrm{SO}(16)$, normalized as $\kappa\left(T_{n}, T_{m}\right)=-2 \delta_{n m}$, and with the identification $f_{n}=2 k_{n} \cdot{ }^{11}$

Let us now come back to the bosonization. The on-shell degrees of freedom of the Fermi multiplets $\Gamma_{n}$ (i.e. discarding the auxiliary fields $G_{n}$ that vanish on-shell), namely left-moving Weyl fermions $\gamma_{n}$, can be embedded into 8 chiral superfields $B_{n}$. After bosonization, $\gamma_{n}$ is associated with the left-moving part of $\operatorname{Im}\left(b_{n}\right)$, the latter being a

\footnotetext{
${ }^{9}$ Naturally, there are instanton solutions associated with the unhatted gauge fields, since the chiral superfields of the base GLSM are minimally coupled to them.

${ }^{10}$ There exists a sort of 'fermionic gauge symmetry', inherited from the decomposition of the $(2,2)$ gauge multiplet into $(0,2)$ multiplets, that can be used to dualize a Fermi multiplet [22], but it is not useful in the present context.

${ }^{11}$ One uses naturally the same integral basis of anti-self-dual two-forms for defining the torus and gauge bundles, corresponding to the intersection matrix $d=\left(-E_{8}\right) \oplus\left(-E_{8}\right) \oplus\left(-2 I_{3}\right)$.
} 
compact boson at radius $R_{f}=1 / \sqrt{2}$ in our conventions. Extra degrees of freedom are of course necessary in order to get full chiral multiplets; however, whenever they will be decoupled from the other degrees of freedom after duality, it will be possible to safely discard these spectator fields from the dual theory.

The original Fermi multiplets generated an anomaly for the unhatted gauge symmetry associated with the torsional GLSM, reading

$$
\delta_{\Xi} \mathcal{L}_{\text {eff }}=-\frac{f_{n} f^{n}}{8} \int \mathrm{d} \theta^{+} \Xi \Upsilon+h . c . .
$$

Obviously, after bosonization, one should reproduce the same gauge variation, however not as a quantum anomaly but as a classical gauge-variance. As for the torus fibration discussed in subsection 2.3, the chiral superfields $B_{n}$ are then coupled both minimally and axially, with a shift charge $\mathfrak{v}_{n}$ and an axial coupling

$$
-\frac{i k_{n}}{4} \int \mathrm{d} \theta^{+} \Upsilon B_{n}+\text { h.c. }
$$

giving the gauge variation

$$
\delta_{\Xi} \mathcal{L}_{g}=\frac{1}{4} k_{n} \mathfrak{v}^{n} \int \mathrm{d} \theta^{+} \Upsilon \Xi+h . c . .
$$

Hence the Bose-Fermi equivalence sets

$$
2 k_{n} \mathfrak{v}^{n}=-f_{n} f^{n} .
$$

On top of this relation, one should keep in mind that, in order to decouple the (noncompact) real part of the $b_{n}$ scalar fields, one should satisfy the relation (see eq. (2.15)):

$$
R_{n}^{2} \mathfrak{v}_{n}+k_{n}=0
$$

which, given that all the bosons are at the fermionic radius $R_{n}=1 / \sqrt{2}$, sets (provided that the same should hold for any choice of $\left\{f_{n}\right\}$ 's)

$$
f_{n}=-\mathfrak{v}_{n}=2 k_{n}
$$

up to an overall sign choice.

At this stage one needs to be careful about the periodicities of the $B_{n}$ 's that are subject to the left-moving GSO projection. One has to consider the identifications

$$
\begin{aligned}
& B_{n} \sim B_{n}+2 i \pi N_{n}, \quad N_{n} \in \mathbb{Z}, \quad \sum_{n=1}^{8} N_{n} \equiv 0 \quad \bmod 2, \\
& B_{n} \sim B_{n}+i \pi N, \quad N \in \mathbb{Z},
\end{aligned}
$$

where the last condition in eq. (4.7a) is imposed by the existence of the Ramond sector, while invariance under $(4.7 \mathrm{~b})$ is a consequence of projecting onto states with even leftmoving worldsheet fermion number. As a consequence, imposing that the path integral - in 
particular the axial coupling (4.2) - should be single-valued under the identifications (4.7) in any instanton sector, singles out two consistent families of line bundles: ${ }^{12}$

$$
\begin{array}{ll}
\vec{k} \in \mathbb{Z}^{8}, & \sum_{n=1}^{8} k_{n} \equiv 0 \bmod 2, \\
\text { or } & \sum_{n=1}^{8} k_{n} \equiv 0 \bmod 2 .
\end{array}
$$

Consistent gauge bundles $V$ in heterotic are constrained by the vanishing of the second Stiefel-Whitney class [43, 44], namely

$$
c_{1}(V) \in H^{2}(\mathcal{S}, 2 \mathbb{Z})
$$

which gives in the present context (taking into account the normalization of the magnetic charges discussed above) a target-space understanding of the condition $\sum_{n=1}^{8} k_{n} \equiv 0$ $\bmod 2$.

\subsection{Wilson lines}

Bosonizing the left-moving fermions belonging to the Fermi multiplets is also useful in describing the Wilson lines that can be added to the $T^{2} \hookrightarrow \mathcal{C}_{6} \rightarrow K 3$ solutions, see eq. (2.5). After bosonization they correspond in the GLSM to off-diagonal couplings whose Lagrangian density is of the form

$$
L_{\mathrm{WL}}=-\frac{i}{4} \alpha_{n}^{a}\left(\Omega_{a}+\bar{\Omega}_{a}+2 \mathfrak{w}_{a} \mathcal{A}\right) \partial_{-}\left(B^{n}-\bar{B}^{n}\right),
$$

where summation over $a=1,2$ and $n=1, \ldots, N$ is implied. Classical gauge invariance requires that

$$
\sum_{n} \alpha_{n}^{a} \mathfrak{v}_{n}=0
$$

whose simplest solution is to have all Fermi multiplets involved being neutral. After refermonization, the expression of eq. (4.10) in components gives couplings like $\bar{\gamma}_{-}^{n} \alpha_{n}^{a}\left[\partial_{-}\left(\omega_{a}-\bar{\omega}_{a}\right)+2 \mathfrak{w}_{a} A_{+}\right] \gamma_{-}^{n}$, corresponding indeed in space-time to a gauge connection of the form (2.5). The other terms in (4.10) give off-diagonal kinetic terms for the non-compact scalar fields $\operatorname{Re}\left(\omega_{a}\right)$ and $\operatorname{Re}\left(b_{n}\right)$ - which eventually decouple from the theory - and for the free worldsheet fermions, hence can be rotated away.

We shall see below that, for special values, the Wilson line moduli $\alpha_{n}^{a}$ can be traded for torus moduli under T-duality.

\footnotetext{
${ }^{12}$ In the $\operatorname{Spin}(32) / \mathbb{Z}_{2}$ theory, these two classes of line bundles would correspond respectively to bundles with our without vector structure [42].
} 


\subsection{Gauge-torus duality}

After these preliminary steps one can move to gauge-torus bundle dualities in $T^{2} \hookrightarrow \mathcal{C}_{6} \rightarrow$ $K 3$ torsional gauged linear sigma models.

For definiteness we choose, as written above, an $E_{8} \times E_{8}$ model such that the nonAbelian component of the gauge bundle (that is part of the $(0,2)$ GLSM with $K 3$ target space) lies in the first $E_{8}$ factor. The second $E_{8}$ factor is chosen to host only an Abelian bundle, corresponding to a set of 8 free Fermi multiplets whose charges are given by a vector $\vec{k}$, belonging to one of the two families (4.8a), (4.8b). Our aim is to find how the $T^{2}$ fibration and the Abelian gauge bundle can mix. As we shall see, this duality only makes sense at specific points in the two-torus discrete moduli space.

To be more specific, we wish to find a perturbative duality exchanging the charges of some bosonized Fermi multiplet $B_{\nu}$ of charge $k_{\nu}$ and, say, $\Omega_{1}$, one of the two chiral multiplets describing the torus fiber over K3, that we take neutral, i.e. with a vanishing shift-charge $\mathfrak{w}_{1}$. In other words, the manifold reduces to a circle bundle over $K 3$ of topological charge $\mathfrak{w}_{2}$ times a circle.

In order to use the technology developed in section 3, we can consider an 'auxiliary' complexified two-torus made with the chiral superfields $\Omega_{1}$ and $B_{\nu}$, whose moduli are set to $v=i$ and $\tau=i / 2$, and whose complex topological charge is $\mathfrak{n}=-i k_{\nu}$. After a $v \rightarrow-1 / v$ duality this model is mapped to a dual model of the same type, with the same torus moduli (crucially, the dual superfield $\tilde{B}_{\nu}$ being still at the fermionic radius), and with the dual charge

$$
\tilde{\mathfrak{n}}=-\bar{v} \mathfrak{n}=k_{\nu} .
$$

Compared to the ordinary two-torus case, there is a subtlety owing to the GSO projection. In deriving the duality from a quotient, we had applied the gauge-fixing condition $B_{\nu}=0$, see around eq. (3.24). However, since this superfield is subject to the identifications (4.7), there exists corresponding residual large gauge transformations that are not fixed in this gauge. It boils down to assigning to $\tilde{\Omega}_{1}$, the superfield dual to $\Omega_{1}$, a shift-charge under the GSO projection. This seems consistent with the analysis of [6], where a detailed analysis of the torsional GLSMs under consideration was done. It was shown there that the shift-superfields of the torus fibration must carry a non-zero shift-charge under the global $\mathrm{U}(1)_{L}$ symmetry which is used in defining the left GSO projections. At the same time, the dual superfield $\tilde{B}_{\nu}$ is expected to inherit the GSO charge assignment of $\Omega_{1}$. It would be useful to study this point in more depth in order to understand its target-space interpretation. ${ }^{13}$

After refermionizing $\tilde{B}_{\nu}$ and throwing away the decoupled extra degrees of freedom belonging to this chiral multiplet, one gets the pullback of an Abelian gauge bundle on K3 whose embedding $\vec{k}$ in the second $E_{8}$ is such that its $\nu$-th component vanishes, and a two-torus principal bundle of topological charge $\mathfrak{w}=k_{\nu}+T \mathfrak{w}_{2}$. Hence T-duality relates two solutions with different topologies.

\footnotetext{
${ }^{13}$ It is worthwhile to remind that the condition (4.9), which plays an important role in this discussion, is necessary as the bundle should admit spinors, which belong to the massive sector of heterotic strings, hence are 'invisible' to supergravity.
} 
In retrospect, looking back at the analysis of line bundles done in subsection 4.1 , the dual model cannot be interpreted as describing a standard geometric $T^{2} \hookrightarrow \mathcal{C}_{6} \rightarrow K 3$ compactification for every value of $k_{\nu}$. As $k_{\nu}$ needs to be integer in order to get a proper geometrical interpretation, only bundles of the type (4.8a) can be chosen as starting points. Furthermore, as we wish that the condition $c_{1}(V) \in H^{2}(\mathcal{S}, 2 \mathbb{Z})$ still holds after the duality, one needs to consider only the cases $k_{\nu} \in 2 \mathbb{Z}$.

The worldsheet theories should still make sense otherwise, since the modified GSO projection that is obtained after duality, see the discussion above, eventually gives consistent models; however they won't have a straightforward geometric interpretation.

Non-orthogonal tori and Wilson lines. As $U_{1}$ and $T_{1}$, the real part of the Kähler and complex structures of the fibered two-torus, are generically non-zero, there are extra non-diagonal terms appearing in the dual theory, that we wish to identify as Wilson lines similar to (4.10). We need to avoid the appearance of terms like $\left(B_{\nu}+\bar{B}_{\nu}\right) \partial_{-}\left(\Omega_{2}-\bar{\Omega}_{2}\right)$, since the real part of $B_{\nu}$ should eventually decouple from the torsional GLSM. This is granted whenever $U_{1}=2 T_{1}$, leading, as $R_{1}^{2}=U_{2} / T_{2}=2$, to

$$
U=2 T
$$

which is a condition for the perturbative duality exchanging the torus fibration and the Abelian gauge bundle to exist. Whenever the consistency condition (4.13) is satisfied, the dual Lagrangian density contains a term

$$
-\frac{i}{4} T_{1}\left(\Omega_{2}+\bar{\Omega}_{2}+2 \mathfrak{w}_{2} \mathcal{A}\right) \partial_{-}\left(B_{\nu}-\bar{B}_{\nu}\right)
$$

hence the gauge bundle of the dual theory embedded in the second $E_{8}$ is the Whitney sum of the pullback of the Abelian gauge bundle over the K3 base discussed above and of a line bundle over the total space $\mathcal{C}_{6}$, given by a Wilson line around the one-cycle of the fiber corresponding to $\Im\left(\omega_{2}\right)$ with $\alpha_{\nu}^{2}=T_{1} \in \mathbb{Q}$.

It is possible to get rid of the remaining $S^{1}$ fibration over the $K 3$ base as well, by further dualizing the model. Other, more complicated examples are naturally possible by considering dualities involving also free Fermi multiplets from the first $E_{8}$ factor, or $\operatorname{Spin}(32) / \mathbb{Z}_{2}$-based constructions.

Wilson line moduli and duality. Perturbative dualities map $T^{2} \hookrightarrow \mathcal{C}_{6} \rightarrow K 3$ compactifications with some HYM gauge bundle to $K 3 \times T^{2}$ compactifications with an extra Abelian gauge bundle. In the former model the torus moduli $(U, T)$ are generically quantized, belonging to the same number field $\mathbb{Q}(\sqrt{D})$, while in the latter model they are free to take any value. The upshot is that the torus moduli are traded for gauge (i.e. Wilson lines) moduli.

To illustrate this point, let us consider the simplest example of gauge/torus duality. The starting point is an $S^{1} \times K 3$ compactification, with a line bundle for which only the component $k_{\nu}$ of the charge vector is non-vanishing. In this model, while the radius of the circle is obviously a free moduli, there are only seven independent Wilson lines moduli from the second $E_{8}$ factor, see the constraint (4.11). The dual model is an $S^{1} \hookrightarrow \mathcal{C}_{5} \rightarrow K 3$ compactification, without gauge bundle in the second $E_{8}$ factor. In this case there are no 
constraints on the Wilson lines, while the radius of the circle fiber is quantized as $R^{2} \in \mathbb{Q}$. Therefore, the dimension of the connected component of the moduli space containing the circle-gauge bundle duality point is the same on both sides, as it should since these are two descriptions of the same physics.

\section{Conclusion}

In this work we have studied perturbative dualities in torsional principal torus bundles compactifications of the heterotic string. Using a gauged linear sigma-model approach allowed us to prove that these dualities, that one can infer from the Buscher rules whose validity in this context is not granted, are exact symmetries of the theory.

The first type of duality maps the torus fiber onto itself and gives a model lying in the same class as the original one, provided that the Kaluza-Klein charges defining the fibration are transformed accordingly. A very interesting outcome is that both moduli of the twotorus, the complex and Kähler structure, should be quantized in a precise way, otherwise duality transformations acting on the Kähler structure wouldn't make sense. While these quantization conditions are rather familiar in the type IIB orientifolds duals, it is interesting to see them arising in a completely different way in the heterotic sigma-model.

The second type of duality, mixing the torus bundle with Abelian gauge bundles, is particularly fascinating since it relates solutions with different topologies. At the gauged linear sigma-model level it maps the torsional GLSMs introduced recently to more familiar ordinary $(0,2)$ GLSMs whose gauge bundle has an Abelian component; hence there isn't any sharp distinction between these two classes of models. It would be interesting to understand better to which extent the interplay of the duality with the generalized left-moving GSO projection that we mentioned in the text could blur the geometrical interpretation of this duality.

Compared to the ordinary $K 3 \times T^{2}$ compactifications, the $T^{2} \hookrightarrow \mathcal{C}_{6} \rightarrow K 3$ solutions have two distinguished features, besides the torus fibration itself. As follows from supersymmetry, the CY base is warped, and there is a non-zero three-form flux. One can wonder what happens to these features under a torus-gauge duality. With a non-compact Eguchi-Hanson base, for which both a worldsheet CFT and a proper supergravity limit are available [32], the answer can be found. The warp factor stays the same, while the NSNS three-form looses its components with a leg along the torus but doesn't vanish. In the compact case, considering $K 3 \times T^{2}$ with a departure from the standard embedding, satisfying the tadpole condition (2.6), a Ricci-flat metric on $K 3$ is a solution of the supersymmetry equations at tree level in the $\alpha^{\prime}$ expansion, while departure from the CY condition as well as torsion are expected to arise at higher order in $\alpha^{\prime}$. The fibered solutions are built upon a very different ansatz, including torsion and warping from the start, but, as the tadpole condition (2.6) forbids the two-torus volume from being large in string units, the distinction between both type of solutions in supergravity, as far as the base is concerned, is not sharply defined.

This duality has also interesting consequences on the effective four-dimensional actions deriving from these compactifications. For instance, the one-loop threshold corrections to the gauge couplings is well-known for $K 3 \times T^{2}$ compactifications with or without Wilson 
lines, being a derived product of the elliptic genus (see e.g. [45] and references therein). Provided that the torus and Wilson line moduli are mapped through the duality according to the rules derived from our analysis, the same quantity gives also the one-loop corrections for compactifications on torsional $T^{2} \hookrightarrow \mathcal{C}_{6} \rightarrow K 3$ manifolds.

Finally, an ambitious goal would be to find similar duality relations between torsional and torsion-free heterotic solutions with $\mathcal{N}=1$ supersymmetry in four dimensions, more specifically between $\mathrm{SU}(3)$-holonomy and $\mathrm{SU}(3)$-structure compactifications. This requires a good handle on $(0,2)$ mirror symmetry since, in this case, worldsheet instantons are expected to play an important role. We leave this analysis for future work.

\section{Acknowledgments}

I would like to thank Luca Carlevaro, Amit Giveon, Stefan Groot-Nibbelink, Nick Halmagyi, Josh Lapan and Jan Troost for enlightening discussions. This work was supported by French state funds managed by the ANR within the Investissements d'Avenir programme under reference ANR-11-IDEX-0004-02.

\section{A Two-dimensional $(0,2)$ superspace}

Minkowskian $(0,2)$ superspace is spanned by the coordinates $\left(x^{+}, x^{-}, \theta^{+}, \bar{\theta}^{+}\right)$with $\bar{\theta}^{+}=$ $\left(\theta^{+}\right)^{\dagger}$. The associated Berezin integral reads (with $\mathrm{d}^{2} \theta=\mathrm{d} \bar{\theta}^{+} \mathrm{d} \theta^{+}$):

$$
\int \mathrm{d}^{2} \theta \theta^{+} \bar{\theta}^{+}=1
$$

We define the super-space derivatives and super-charges as follows:

$$
\begin{array}{ll}
\mathcal{Q}_{+}=\partial_{\theta^{+}}+i \bar{\theta}^{+} \partial_{+}, & \overline{\mathcal{Q}}_{+}=-\partial_{\bar{\theta}^{+}}-i \theta^{+} \partial_{+}, \\
D_{+}=\partial_{\theta^{+}}-i \bar{\theta}^{+} \partial_{+}, & \bar{D}_{+}=-\partial_{\bar{\theta}^{+}}+i \theta^{+} \partial_{+} .
\end{array}
$$

The non-trivial anti-commutators are then

$$
\left\{\bar{D}_{+}, D_{+}\right\}=2 i \partial_{+}, \quad\left\{\overline{\mathcal{Q}}_{+}, \mathcal{Q}_{+}\right\}=-2 i \partial_{+}
$$

\section{A.1 Superfields}

We give the component expansion of the superfields that are needed in this work.

Chiral superfields. are defined by the constraint

$$
\bar{D}_{+} \Phi=0
$$

Hence the chiral superfield reads in components

$$
\Phi=\phi+\sqrt{2} \theta^{+} \lambda_{+}-i \theta^{+} \bar{\theta}^{+} \partial_{+} \phi
$$


Fermi superfields. have as a bottom component a left-moving fermion. They satisfy generically the constraint

$$
\bar{D}_{+} \Gamma=\sqrt{2} E(\Phi),
$$

where $E$ is an holomorphic function. Hence have the component expansion

$$
\Gamma=\gamma_{-}+\sqrt{2} \theta^{+} G-\sqrt{2} \bar{\theta}^{+} E(\Phi)-i \theta^{+} \bar{\theta}^{+} \partial_{+} \gamma_{-},
$$

where $G$ is an auxiliary field.

Gauge multiplets. are actually described by a pair of $(0,2)$ superfields, namely $\mathcal{A}$ and $\mathcal{V}$. Super-gauge transformations act as

$$
\mathcal{A} \rightarrow \mathcal{A}+\frac{i}{2}(\bar{\Xi}-\Xi), \quad \mathcal{V} \rightarrow \mathcal{V}-\frac{1}{2} \partial_{-}(\Xi+\bar{\Xi})
$$

where $\Xi$ is a chiral superfield.

In the Wess-Zumino gauge things get simpler, even though one should be careful while dealing with classically non gauge-invariant actions. The residual gauge symmetry is given in this gauge by

$$
\Xi=\rho-i \theta^{+} \bar{\theta}^{+} \partial_{+} \rho
$$

with real $\rho$, while the component expansion of $\mathcal{A}$ and $\mathcal{V}$ read

$$
\begin{aligned}
& \mathcal{A}=\theta^{+} \bar{\theta}^{+} A_{+} \\
& \mathcal{V}=A_{-}-2 i \theta^{+} \bar{\mu}_{-}-2 i \bar{\theta}^{+} \mu_{-}+2 \theta^{+} \bar{\theta}^{+} D
\end{aligned}
$$

where $D$ is an auxiliary field. Accordingly the components $A_{ \pm}=A_{0} \pm A_{1}$ of the gauge field are shifted under the residual gauge transformations as

$$
A_{ \pm} \stackrel{\rho}{\longrightarrow} A_{ \pm}-\partial_{ \pm} \rho
$$

In order to couple the gauge superfields to the chiral and Fermi superfields we first define ordinary covariant derivatives as

$$
\nabla_{ \pm}=\partial_{ \pm}+i Q A_{ \pm}
$$

Then we construct the gauge-covariant superderivatives as follows:

$$
\begin{aligned}
& \mathfrak{D}_{+}=\left(\partial_{\theta^{+}}-i \bar{\theta}^{+} \nabla_{+}\right)=D_{+}+Q \bar{\theta}^{+} A_{+} \\
& \overline{\mathfrak{D}}_{+}=\left(-\partial_{\bar{\theta}^{+}}+i \theta^{+} \nabla_{+}\right)=\bar{D}_{+}-Q \theta^{+} A_{+}
\end{aligned}
$$

They satisfy the algebra

$$
\left\{\mathfrak{D}_{+}, \overline{\mathfrak{D}}_{+}\right\}=2 i \nabla_{+} \cdot
$$

We also need the superspace version of the gauge-covariant derivative, since our theory is chiral. It is defined as

$$
\mathcal{D}_{-}=\partial_{-}+i Q \mathcal{V}
$$

whose lowest component is $\nabla_{-}$. 
Charged matter multiplets. A chiral multiplet of charge $Q$ needs to satisfy the gaugecovariant constraint:

$$
\overline{\mathfrak{D}}_{+} \Phi=0
$$

which is solved by

$$
\Phi=\phi+\sqrt{2} \theta^{+} \lambda_{+}-i \theta^{+} \bar{\theta}^{+} \nabla_{+} \phi
$$

In other words, since

$$
\overline{\mathfrak{D}}_{+}=e^{Q \mathcal{A}} \bar{D}_{+} e^{-Q \mathcal{A}}
$$

We have that

$$
\Phi=e^{Q \mathcal{A}} \Phi_{0}
$$

where $\Phi_{0}$ is a superfield obeying the standard chirality constraint $\bar{D}_{+} \Phi_{0}=0$.

Similarly, a charged Fermi superfield of charge $q$ can be obtained as $\Gamma=e^{q \mathcal{A}} \Gamma_{0}$ where $\Gamma_{0}$ satisfies $\bar{D}_{+} \Gamma_{0}=\sqrt{2} E$. Hence the superfield $\Gamma$ has the component expansion:

$$
\Gamma=\gamma_{-}+\sqrt{2} \theta^{+} G-\sqrt{2} \bar{\theta}^{+} E(\Phi)-i \theta^{+} \bar{\theta}^{+} \nabla_{+} \gamma_{-},
$$

where as before $E$ is an holomorphic function in the chiral superfields.

Shift chiral superfield. We define a shift chiral superfield $\Omega$, which is a chiral superfield which transforms under gauge transformations as

$$
\Omega \stackrel{\Xi}{\longrightarrow} \Omega+i \mathfrak{w} \Xi
$$

The components expansion of this superfield is simply

$$
\Omega=\omega+\sqrt{2} \theta^{+} \chi_{+}-i \theta^{+} \bar{\theta}^{+} \partial_{+} \omega
$$

such that it satisfies the standard chirality constraint. Notice that only the imaginary part of $\Omega$ is shifted by the gauge transformation (A.21).

\section{A.2 (0,2) Lagrangians}

Let us start with the kinetic term for a chiral field $\Phi$ of charge $Q$, whose components expansion is given by (A.17). It is given by

$$
\begin{aligned}
\mathcal{L}_{s} & =-\frac{i}{2} \int \mathrm{d}^{2} \theta^{+} \bar{\Phi} \mathcal{D}_{-} \Phi \\
& =\frac{1}{2}\left(\overline{\nabla_{+} \phi} \nabla_{-} \phi+\nabla_{-} \bar{\phi} \nabla_{+} \phi\right)+i \bar{\lambda}_{+} \nabla_{-} \lambda_{+}+i \sqrt{2} Q\left(\lambda_{+} \mu_{-} \bar{\phi}+h . c .\right)+Q \phi \bar{\phi} D .
\end{aligned}
$$

Let us now move on to the case of a Fermi superfield of charge $q$. One has the following component expansion:

$$
\begin{aligned}
\mathcal{L}_{f} & =-\frac{1}{2} \int \mathrm{d}^{2} \theta^{+} \bar{\Gamma} \Gamma \\
& =i \bar{\gamma}_{-} \nabla_{+} \gamma_{-}+|G|^{2}-|E(\phi)|^{2}-\left(E^{\prime}(\phi) \bar{\gamma}_{-} \lambda_{+}+\text {h.c. }\right)
\end{aligned}
$$


The gauge kinetic term is written in terms of the field strength superfield, which is a chiral superfield

$$
\Upsilon=\bar{D}_{+}\left(\partial_{-} \mathcal{A}+i \mathcal{V}\right)=-2\left(\mu_{-}-i \theta^{+}\left(D-i F_{01}\right)-i \theta^{+} \bar{\theta}^{+} \partial_{+} \mu_{-}\right)
$$

with $2 F_{01}=\partial_{-} A_{+}-\partial_{+} A_{-}$. Including a possible constant FI term, of parameter $t=i r+\frac{\theta}{2 \pi}$, one has

$$
\begin{aligned}
\mathcal{L}_{g} & =-\frac{1}{8 e^{2}} \int \mathrm{d}^{2} \theta^{+} \bar{\Upsilon} \Upsilon+\frac{t}{4} \int \mathrm{d} \theta^{+} \Upsilon+\text { h.c. } \\
& =\frac{1}{2 e^{2}}\left(2 i \bar{\mu}_{-} \partial_{+} \mu_{-}+D^{2}+F_{01}^{2}\right)-r D+\frac{\theta}{2 \pi} F_{01}
\end{aligned}
$$

Due to the presence of chiral fermions there is generically a gauge anomaly. It is given as

$$
\delta_{\Xi} W=\frac{\mathfrak{A}}{16 \pi} \int \mathrm{d}^{2} x \int \mathrm{d} \theta^{+} \Xi \Upsilon+\text { h.c. },
$$

with $\mathfrak{A}=Q_{i} Q^{i}-q_{n} q^{n}$.

Finally, a gauge-invariant kinetic term for the shift chiral superfield can be constructed in superspace as follows

$$
\begin{aligned}
\mathcal{L}_{s}= & -\frac{i}{4} \int \mathrm{d}^{2} \theta(\Omega+\bar{\Omega}+2 m \mathcal{A})\left(\partial_{-}(\Omega-\bar{\Omega})+2 i \mathfrak{w} \mathcal{V}\right) \\
= & \frac{1}{4} \partial_{+}(\omega+\bar{\omega}) \partial_{-}(\omega+\bar{\omega})-\frac{1}{4}\left(\partial_{+}(\omega-\bar{\omega})+2 i m A_{+}\right)\left(\partial_{-}(\omega-\bar{\omega})+2 i m A_{-}\right) \\
& +\frac{i}{2}\left(\chi_{+} \partial_{-} \bar{\chi}_{+}+\bar{\chi}_{+} \partial_{-} \chi_{+}\right)+i \sqrt{2} m\left(\chi_{+} \mu_{-}+\bar{\chi}_{+} \bar{\mu}_{-}\right)+m D(\omega+\bar{\omega}) .
\end{aligned}
$$

It is possible to add a FI-like term (axial coupling):

$$
\begin{aligned}
\mathcal{L}_{g} & =-\frac{i h}{4} \int \mathrm{d} \theta^{+} \Omega \Upsilon+h . c . \\
& =h D \operatorname{Re}(\omega)+h F_{01} \operatorname{Im}(\omega)-\frac{i h}{\sqrt{2}}\left(\mu_{-} \chi_{+}-\bar{\mu}_{-} \bar{\chi}_{+}\right)
\end{aligned}
$$

Whenever the shift superfield is charged, this coupling is classically not gauge invariant. Indeed

$$
\delta_{\Xi} \mathcal{L}_{g}=\frac{h}{4} \mathfrak{w} \int \mathrm{d} \theta^{+} \Xi \Upsilon+h . c .
$$

This terms combines nicely with the one-loop anomaly, eq. (A.27) in order to get a gauge-invariant theory. The axial coupling can also be rewritten, in order to facilitate the duality transformations, as an integral over the whole superspace, using eq. (A.25):

$$
\mathcal{L}_{g}=\frac{h}{4} \int \mathrm{d}^{2} \theta^{+}\left[\mathcal{V}(\Omega+\bar{\Omega})+i \mathcal{A} \partial_{-}(\Omega-\bar{\Omega})\right]+t . d . .
$$

Notice that one could also consider an ordinary charged chiral superfield $\Phi$, with a logarithmic FI-like term, leading to similar effects:

$$
\mathcal{L}_{g}^{\prime}=-\frac{i h}{4} \int \mathrm{d} \theta^{+} \Upsilon \log \tilde{\Phi}+h . c .
$$


Superpotential terms. The last term in the GLSM is the superpotential term, given by a set of holomorphic functions $J$ of the chiral superfields. It has to satisfy the constraint $E^{a} J_{a}=0$ (where $a$ runs over the Fermi superfields). It reads

$$
\begin{aligned}
\mathcal{L}_{p} & =-\frac{\mu}{2} \int \mathrm{d} \theta^{+} \Gamma J+h . c . \\
& =\frac{\mu}{\sqrt{2}}(G J+h . c .)+\frac{\mu}{\sqrt{2}} \gamma_{-}\left(\lambda_{+} \partial_{\phi} J+\chi_{+} \partial_{\omega} J\right)+\text { h.c. }
\end{aligned}
$$

After solving for the auxiliary fields of the full theory one gets the scalar potential

$$
U(\phi, \omega)=\frac{e^{2}}{8}\left(Q|\phi|^{2}-r\right)^{2}+\frac{\mu}{2}|J|^{2}+|E|^{2},
$$

which defines the vacua of the theory $Q|\phi|^{2}=r, J=0$ and $E=0$ modulo gauge transformations.

\section{B Two-tori}

The moduli space of $T^{2}$ compactifications is spanned by the vacuum expectation values of two complex fields

$$
T=\frac{G_{12}+i \sqrt{\operatorname{det} G}}{G_{11}}, \quad U=B_{12}+i \sqrt{\operatorname{det} G}
$$

where the coordinates $\left(x^{1}, x^{2}\right)$ are $2 \pi$-periodic. In terms of these parameters the metric is rewritten conveniently as

$$
\mathrm{d} s^{2}=\frac{U_{2}}{T_{2}}\left|\mathrm{~d} x^{1}+T \mathrm{~d} x^{2}\right|^{2}
$$

It is natural to gather the metric and the B-field in a single matrix

$$
E=G+B=\frac{U_{2}}{T_{2}}\left(\begin{array}{cc}
1 & T_{1}+T_{2} U_{1} / U_{2} \\
T_{1}-T_{2} U_{1} / U_{2} & T \bar{T}
\end{array}\right)
$$

String compactifications on a two-torus have a common $O(2,2, \mathbb{Z})$ perturbative duality group which decomposes as

$$
\operatorname{PSL}(2, \mathbb{Z}) \times \operatorname{PSL}(2, \mathbb{Z}) \times \mathbb{Z}_{2} \times \mathbb{Z}_{2}
$$

The first $\operatorname{PSL}(2, \mathbb{Z})$ acts on $T$ as

$$
T \rightarrow \frac{a T+b}{c T+d}, \quad a d-b c=1, \quad a, b, c, d \in \mathbb{Z}
$$

and leaves $U$ invariant. It corresponds to the familiar modular group of the two-torus (which does not contain any 'stringy' symmetry).

The second $\operatorname{PSL}(2, \mathbb{Z})$ acts on $U$ in a similar way, leaving $T$ invariant; it contains e.g. integer shifts of the $B$-field. It generalizes the usual T-duality of $S^{1}$ compactifications.

The extra $\mathbb{Z}_{2}$ symmetries are the spacetime parity $(U, T) \rightarrow(-\bar{U},-\bar{T})$ and T-duality along $x^{1}$, which exchanges the generalized Kähler modulus and the complex structure

$$
(T ; U) \stackrel{T_{x}}{\longrightarrow}(U ; T),
$$

hence is nothing but mirror symmetry in one complex dimension. 
Open Access. This article is distributed under the terms of the Creative Commons Attribution License which permits any use, distribution and reproduction in any medium, provided the original author(s) and source are credited.

\section{References}

[1] E. Witten, Phases of $N=2$ theories in two-dimensions, Nucl. Phys. B 403 (1993) 159 [hep-th/9301042] [INSPIRE].

[2] J. McOrist, The revival of $(0,2)$ linear $\sigma$-models, Int. J. Mod. Phys. A 26 (2011) 1 [arXiv: 1010.4667] [INSPIRE].

[3] A. Adams, M. Ernebjerg and J.M. Lapan, Linear models for flux vacua, Adv. Theor. Math. Phys. 12 (2008) 817 [hep-th/0611084] [INSPIRE].

[4] J.M. Lapan, Towards the massless spectrum of non-Kähler heterotic compactifications, Nucl. Phys. Proc. Suppl. 171 (2007) 290 [inSPIRE].

[5] A. Adams and D. Guarrera, Heterotic flux vacua from hybrid linear models, arXiv:0902.4440 [INSPIRE].

[6] A. Adams and J.M. Lapan, Computing the spectrum of a heterotic flux vacuum, JHEP 03 (2011) 045 [arXiv: 0908.4294] [INSPIRE].

[7] S. Groot Nibbelink, Heterotic orbifold resolutions as $(2,0)$ gauged linear $\sigma$-models, Fortsch. Phys. 59 (2011) 454 [arXiv: 1012.3350] [INSPIRE].

[8] C. Quigley and S. Sethi, Linear $\sigma$-models with torsion, JHEP 11 (2011) 034 [arXiv: 1107.0714] [INSPIRE].

[9] M. Blaszczyk, S. Groot Nibbelink and F. Ruehle, Green-Schwarz mechanism in heterotic $(2,0)$ gauged linear $\sigma$-models: torsion and NS5 branes, JHEP 08 (2011) 083 [arXiv:1107.0320] [INSPIRE].

[10] C. Quigley, S. Sethi and M. Stern, Novel branches of $(0,2)$ theories, JHEP 09 (2012) 064 [arXiv: 1206.3228] [INSPIRE].

[11] A. Adams, E. Dyer and J. Lee, GLSMs for non-Kähler geometries, JHEP 01 (2013) 044 [arXiv: 1206.5815] [INSPIRE].

[12] I.V. Melnikov, C. Quigley, S. Sethi and M. Stern, Target spaces from chiral gauge theories, JHEP 02 (2013) 111 [arXiv:1212.1212] [INSPIRE].

[13] A. Strominger, Superstrings with torsion, Nucl. Phys. B 274 (1986) 253 [INSPIRE].

[14] K. Dasgupta, G. Rajesh and S. Sethi, $M$ theory, orientifolds and $G$-flux, JHEP 08 (1999) 023 [hep-th/9908088] [INSPIRE].

[15] E. Goldstein and S. Prokushkin, Geometric model for complex non-Kähler manifolds with SU(3) structure, Commun. Math. Phys. 251 (2004) 65 [hep-th/0212307] [INSPIRE].

[16] J.-X. Fu and S.-T. Yau, The theory of superstring with flux on non-Kähler manifolds and the complex Monge-Ampere equation, J. Diff. Geom. 78 (2009) 369 [hep-th/0604063] [INSPIRE].

[17] S. Ivanov and G. Papadopoulos, Vanishing theorems and string backgrounds, Class. Quant. Grav. 18 (2001) 1089 [math/0010038] [INSPIRE].

[18] G. Lopes Cardoso et al., Non-Kähler string backgrounds and their five torsion classes, Nucl. Phys. B 652 (2003) 5 [hep-th/0211118] [InSPIRE]. 
[19] L. Carlevaro and D. Israel, Heterotic resolved conifolds with torsion, from supergravity to CFT, JHEP 01 (2010) 083 [arXiv:0910.3190] [INSPIRE].

[20] F. Chen, K. Dasgupta, J.M. Lapan, J. Seo and R. Tatar, Gauge/gravity duality in heterotic string theory, Phys. Rev. D 88 (2013) 066003 [arXiv:1303.4750] [INSPIRE].

[21] K. Hori and C. Vafa, Mirror symmetry, hep-th/0002222 [INSPIRE].

[22] A. Adams, A. Basu and S. Sethi, (0,2) duality, Adv. Theor. Math. Phys. 7 (2004) 865 [hep-th/0309226] [INSPIRE].

[23] I. Melnikov, S. Sethi and E. Sharpe, Recent developments in (0,2) mirror symmetry, SIGMA 8 (2012) 068 [arXiv:1209.1134] [INSPIRE].

[24] J. Evslin and R. Minasian, Topology change from (heterotic) Narain T-duality, Nucl. Phys. B 820 (2009) 213 [arXiv:0811.3866] [InSPIRE].

[25] D. Andriot, R. Minasian and M. Petrini, Flux backgrounds from twists, JHEP 12 (2009) 028 [arXiv:0903.0633] [INSPIRE].

[26] D. Martelli and J. Sparks, Non-Kähler heterotic rotations, Adv. Theor. Math. Phys. 15 (2011) 131 [arXiv:1010.4031] [INSPIRE].

[27] A. Giveon, M. Porrati and E. Rabinovici, Target space duality in string theory, Phys. Rept. 244 (1994) 77 [hep-th/9401139] [InSPIRE].

[28] M. Becker, L.-S. Tseng and S.-T. Yau, Heterotic Kähler/non-Kähler transitions, Adv. Theor. Math. Phys. 12 (2008) [arXiv:0706.4290] [INSPIRE].

[29] S. Sethi, A note on heterotic dualities via M-theory, Phys. Lett. B 659 (2008) 385 [arXiv:0707.0295] [INSPIRE].

[30] D. Andriot, Heterotic string from a higher dimensional perspective, Nucl. Phys. B 855 (2012) 222 [arXiv:1102.1434] [InSPIRE].

[31] M. Roček and E.P. Verlinde, Duality, quotients and currents, Nucl. Phys. B 373 (1992) 630 [hep-th/9110053] [INSPIRE].

[32] L. Carlevaro, D. Israel and P.M. Petropoulos, Double-scaling limit of heterotic bundles and dynamical deformation in CFT, Nucl. Phys. B 827 (2010) 503 [arXiv:0812.3391] [INSPIRE].

[33] L.B. Anderson, J. Gray, A. Lukas and E. Palti, Two hundred heterotic Standard Models on smooth Calabi-Yau threefolds, Phys. Rev. D 84 (2011) 106005 [arXiv:1106.4804] [INSPIRE].

[34] M. Blaszczyk, S. Groot Nibbelink and F. Ruehle, Gauged linear $\sigma$-models for toroidal orbifold resolutions, JHEP 05 (2012) 053 [arXiv:1111.5852] [INSPIRE].

[35] K. Becker, C. Bertinato, Y.-C. Chung and G. Guo, Supersymmetry breaking, heterotic strings and fluxes, Nucl. Phys. B 823 (2009) 428 [arXiv:0904.2932] [InSPIRE].

[36] K. Becker and S. Sethi, Torsional heterotic geometries, Nucl. Phys. B 820 (2009) 1 [arXiv:0903.3769] [INSPIRE].

[37] J. Held, D. Lüst, F. Marchesano and L. Martucci, DWSB in heterotic flux compactifications, JHEP 06 (2010) 090 [arXiv: 1004.0867] [INSPIRE].

[38] G.W. Moore, Les Houches lectures on strings and arithmetic, hep-th/0401049 [INSPIRE].

[39] S. Gukov and C. Vafa, Rational conformal field theories and complex multiplication, Commun. Math. Phys. 246 (2004) 181 [hep-th/0203213] [InSPIRE]. 
[40] D. Israel and L. Carlevaro, Local models of heterotic flux vacua: spacetime and worldsheet aspects, Fortsch. Phys. 59 (2011) 716 [arXiv:1109.1534] [INSPIRE].

[41] P.K. Tripathy and S.P. Trivedi, Compactification with flux on K3 and tori, JHEP 03 (2003) 028 [hep-th/0301139] [INSPIRE].

[42] M. Berkooz et al., Anomalies, dualities and topology of $D=6 \mathrm{~N}=1$ superstring vacua, Nucl. Phys. B 475 (1996) 115 [hep-th/9605184] [INSPIRE].

[43] E. Witten, Global anomalies in string theory, to appear in Proc. of Argonne Symp. on Geometry, Anomalies and Topology, Argonne IL U.S.A. March 28-30 1985 [INSPIRE]

[44] D. Freed, Determinants, torsion and strings, Commun. Math. Phys. 107 (1986) 483 [INSPIRE].

[45] P.G. Camara and E. Dudas, Multi-instanton and string loop corrections in toroidal orbifold models, JHEP 08 (2008) 069 [arXiv:0806.3102] [INSPIRE]. 